\title{
Multiwavelength Observations of the Blazar BL Lacertae: A New Fast TeV Gamma-Ray Flare
}

A. U. Abeysekara ${ }^{1}$, W. Benbow ${ }^{2}$ (1), R. Bird ${ }^{3}$ (1), T. Brantseg ${ }^{4}$, R. Brose, ${ }^{5,6}$, M. Buchovecky ${ }^{3}$, J. H. Buckley ${ }^{7}$, V. Bugaev ${ }^{7}$,

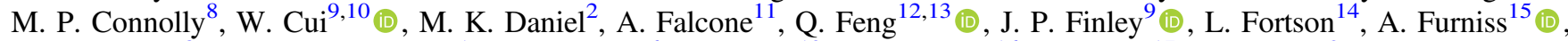
G. H. Gillanders ${ }^{8}$ (1) I. Gunawardhana ${ }^{1}$ (10), M. Hütten ${ }^{6}$, D. Hanna ${ }^{12}$ (1) , O. Hervet ${ }^{16}$, J. Holder ${ }^{17}$, G. Hughes ${ }^{2}$, T. B. Humensky ${ }^{13}$,

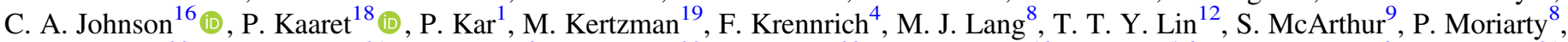

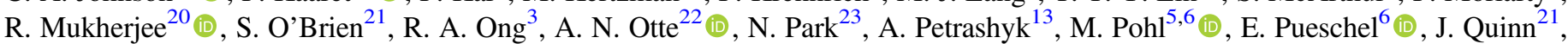
K. Ragan ${ }^{12}$, P. T. Reynolds ${ }^{24}$, G. T. Richards ${ }^{22}$ (1), E. Roache ${ }^{2}$, C. Rulten ${ }^{14}$, I. Sadeh ${ }^{6}$, M. Santander ${ }^{20,25}$, G. H. Sembroski ${ }^{9}$, K. Shahinyan ${ }^{14}$ (D) , S. P. Wakely ${ }^{23}$, A. Weinstein ${ }^{4}$, R. M. Wells ${ }^{4}$, P. Wilcox ${ }^{18}$, D. A. Williams ${ }^{16}$, B. Zitzer ${ }^{12}$

\section{(The VERITAS Collaboration),}

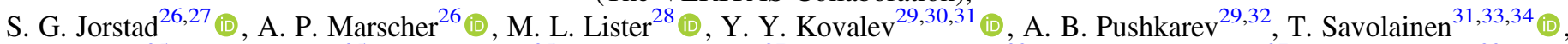
I. Agudo $^{35}$, S. N. Molina ${ }^{35}$, J. L. Gómez ${ }^{35}$, V. M. Larionov ${ }^{27}$ (D) G. A. Borman ${ }^{32}$, A. A. Mokrushina ${ }^{27}$, M. Tornikoski $^{33}$ (iD, A. Lähteenmäki ${ }^{33,34,36}$, W. Chamani ${ }^{33,34}$, S. Enestam ${ }^{33,34}$, S. Kiehlmann ${ }^{37}$, T. Hovatta ${ }^{38}$ (D) P. S. Smith ${ }^{39}$, and P. Pontrelli ${ }^{16}$ ${ }^{1}$ Department of Physics and Astronomy, University of Utah, Salt Lake City, UT 84112, USA

${ }^{2}$ Fred Lawrence Whipple Observatory, Harvard-Smithsonian Center for Astrophysics, Amado, AZ 85645, USA

${ }^{3}$ Department of Physics and Astronomy, University of California, Los Angeles, CA 90095, USA

${ }^{4}$ Department of Physics and Astronomy, Iowa State University, Ames, IA 50011, USA

${ }^{5}$ Institute of Physics and Astronomy, University of Potsdam, D-14476 Potsdam-Golm, Germany ${ }^{6}$ DESY, Platanenallee 6, D-15738 Zeuthen, Germany

${ }^{7}$ Department of Physics, Washington University, St. Louis, MO 63130, USA

${ }^{8}$ School of Physics, National University of Ireland Galway, University Road, Galway, Ireland

${ }^{9}$ Department of Physics and Astronomy, Purdue University, West Lafayette, IN 47907, USA

${ }^{10}$ Department of Physics and Center for Astrophysics, Tsinghua University, Beijing 100084, People's Republic of China

${ }^{11}$ Department of Astronomy and Astrophysics, 525 Davey Lab, Pennsylvania State University, University Park, PA 16802, USA

${ }^{12}$ Physics Department, McGill University, Montreal, QC H3A 2T8, Canada; qifeng@ @evis.columbia.edu

${ }^{13}$ Physics Department, Columbia University, New York, NY 10027, USA

${ }^{14}$ School of Physics and Astronomy, University of Minnesota, Minneapolis, MN 55455, USA

${ }^{15}$ Department of Physics, California State University-East Bay, Hayward, CA 94542, USA

${ }^{16}$ Santa Cruz Institute for Particle Physics and Department of Physics, University of California, Santa Cruz, CA 95064, USA; ohervet@ucsc.edu

${ }^{17}$ Department of Physics and Astronomy and the Bartol Research Institute, University of Delaware, Newark, DE 19716, USA

${ }^{18}$ Department of Physics and Astronomy, University of Iowa, Van Allen Hall, Iowa City, IA 52242, USA

${ }^{19}$ Department of Physics and Astronomy, DePauw University, Greencastle, IN 46135-0037, USA

${ }^{20}$ Department of Physics and Astronomy, Barnard College, Columbia University, NY 10027, USA

${ }^{21}$ School of Physics, University College Dublin, Belfield, Dublin 4, Ireland

${ }^{22}$ School of Physics and Center for Relativistic Astrophysics, Georgia Institute of Technology, 837 State Street NW, Atlanta, GA 30332-0430, USA

${ }^{23}$ Enrico Fermi Institute, University of Chicago, Chicago, IL 60637, USA

${ }^{24}$ Department of Physical Sciences, Cork Institute of Technology, Bishopstown, Cork, Ireland

${ }^{25}$ Department of Physics and Astronomy, University of Alabama, Tuscaloosa, AL 35487, USA

${ }^{26}$ Institute for Astrophysical Research, Boston University, 725 Commonwealth Avenue, Boston, MA 02215, USA; jorstad@bu.edu

${ }^{27}$ Astronomical Institute, St.Petersburg State University, Universitetskij Pr. 28, Petrodvorets, 198504 St.Petersburg, Russia

${ }^{28}$ Purdue University, 525 Northwestern Avenue, West Lafayette, IN 47907, USA

${ }^{29}$ Astro Space Center of Lebedev Physical Institute, Profsoyuznaya 84/32, 117997 Moscow, Russia

${ }^{30}$ Moscow Institute of Physics and Technology, Dolgoprudny, Institutsky per., 9, Moscow region, 141700, Russia

${ }^{31}$ Max-Planck-Institut für Radioastronomie, Auf dem Hügel 69, D-53121 Bonn, Germany

${ }^{32}$ Crimean Astrophysical Observatory, 98409 Nauchny, Crimea, Russia ${ }^{\dagger}$

${ }^{33}$ Aalto University Metsähovi Radio Observatory, Metsähovintie 114, FI-02540 Kylmälä, Finland

${ }^{34}$ Aalto University Department of Electronics and Nanoengineering, PL 15500, FI-00076 Aalto, Finland

${ }^{35}$ Instituto de Astrofísica de Andalucía (CSIC), Apartado 3004, E-18080 Granada, Spain

${ }^{36}$ Tartu Observatory Observatooriumi 161602 Tõravere, Estonia

${ }^{37}$ Owens Valley Radio Observatory, California Institute of Technology, Pasadena, CA 91125, USA

${ }^{38}$ Tuorla Observatory, Department of Physics and Astronomy, University of Turku, Väisäläntie 20, 21500 Kaarina, Finland

${ }^{39}$ Steward Observatory, University of Arizona, Tucson, AZ 85716, USA

Received 2017 October 31; revised 2018 February 21; accepted 2018 February 26; published 2018 March 28

\begin{abstract}
Combined with measurements made by very-long-baseline interferometry, the observations of fast $\mathrm{TeV}$ gamma-ray flares probe the structure and emission mechanism of blazar jets. However, only a handful of such flares have been detected to date, and only within the last few years have these flares been observed from lower-frequency-peaked BL Lac objects and flat-spectrum radio quasars. We report on a fast $\mathrm{TeV}$ gamma-ray flare from the blazar BL Lacertae observed by the Very Energetic Radiation Imaging Telescope Array System (VERITAS). with a rise time of $\sim 2.3 \mathrm{hr}$ and a decay time of $\sim 36 \mathrm{~min}$. The peak flux above $200 \mathrm{GeV}$ is $(4.2 \pm 0.6) \times 10^{-6}$ photon m $\mathrm{m}^{-2} \mathrm{~s}^{-1}$
\end{abstract}

\footnotetext{
While the AAS journals adhere to and respect UN resolutions regarding the designations of territories (available at http://www.un.org/press/en), it is our policy to use the affiliations provided by our authors on published articles.
} 
measured with a 4-minute-binned light curve, corresponding to $\sim 180 \%$ of the flux that is observed from the Crab Nebula above the same energy threshold. Variability contemporaneous with the $\mathrm{TeV}$ gamma-ray flare was observed in GeV gamma-ray, X-ray, and optical flux, as well as in optical and radio polarization. Additionally, a possible moving emission feature with superluminal apparent velocity was identified in Very Long Baseline Array observations at $43 \mathrm{GHz}$, potentially passing the radio core of the jet around the time of the gamma-ray flare. We discuss the constraints on the size, Lorentz factor, and location of the emitting region of the flare, and the interpretations with several theoretical models that invoke relativistic plasma passing stationary shocks.

Key words: BL Lacertae objects: individual (BL Lacertae $=$ VER J2202+422) - galaxies: active

\section{Introduction}

BL Lac objects belong to a subclass of radio-loud active galactic nuclei (AGNs) known as blazars. They are characterized by featureless optical spectra, non-thermal broadband spectra, and rapid variability, which jointly suggest that their emission originates in relativistic jets closely aligned to our line of sight (e.g., Blandford \& Rees 1978, and references therein).

Fast variability at very high energies $\left(100 \mathrm{GeV} \lesssim E_{\gamma} \lesssim\right.$ $100 \mathrm{TeV}$; VHE), with timescales as short as a few minutes, has been observed in several blazars (e.g., Gaidos et al. 1996; Aharonian et al. 2007; Albert et al. 2007b; Aleksić et al. 2011), including the prototypical BL Lacertae (VER J2202+422; Arlen et al. 2013) located at redshift $z=0.069$ (Miller \& Hawley 1977). Long-term monitoring of BL Lacertae has led to no detection of the source in the $\mathrm{TeV}$ gamma-ray band by the current generation of instruments except during flaring episodes, when its flux has been observed to reach $>100 \%$ of the Crab Nebula flux (C.U.) above $1 \mathrm{TeV}$ in 1998 (Neshpor et al. 2001), $\sim 0.03$ C.U. above $200 \mathrm{GeV}$ in 2005 (Albert et al. 2007a), and most recently $\sim 1.25$ C.U. above $200 \mathrm{GeV}$ with a short variability timescale of $13 \pm 4$ minutes in 2011 (Arlen et al. 2013).

The rapid gamma-ray variability observed in $\mathrm{TeV}$ blazars implies very compact emitting regions, as well as low gammaray attenuation by pair production on infrared/optical photons near the emission zone. While a one-zone synchrotron selfCompton (SSC) model, one of the simplest blazar models (e.g., Ghisellini et al. 1998; Böttcher \& Chiang 2002), has been effective at explaining emission from high-frequency-peaked BL Lac (HBL) objects, the intrinsic pair-production opacity of a relativistic emission zone in such a model depends on its size and Doppler factor, and on the density of lower-energy photons. Therefore, if the synchrotron photons are the main source of the lower-energy radiation, the emitting region must have a small size and/or a large Doppler factor so that the gamma-rays can escape pair production. Alternatively, if an external photon field (e.g., the broad-line region; BLR) dominates the lower-energy radiation, it can cause substantial gamma-ray absorption. As a result, the emitting region is generally expected to be far away from the central region of the AGN, especially for flat-spectrum radio quasars (FSRQs) whose broad-line emission is relatively strong.

BL Lacertae was first classified as a low-frequency-peaked BL Lac (LBL) object because the synchrotron peak frequency of its spectral energy distribution (SED) was measured to be $2.2 \times 10^{14} \mathrm{~Hz}$ (Sambruna et al. 1999), but it was later reclassified as an intermediate-frequency-peaked $\mathrm{BL}$ Lac (IBL) object (Ackermann et al. 2011). It has been reported that the SEDs of several IBLs/LBLs cannot be well described by a one-zone SSC model (see Hervet et al. 2015, and references therein), and more complex models such as multi-zone SSC models or external-radiation Compton (ERC) models are needed.

The large Doppler factor and/or distant downstream emitting region required by the observed fast $\mathrm{TeV}$ variability of blazars, together with the knotty jet structures (both moving and stationary) identified with high-resolution radio observations (e.g., Cohen et al. 2014), can be explained consistently by theoretical models with multiple emitting zones that are either spatially or temporally separated, e.g., structured jets (Ghisellini et al. 2005), jet deceleration (Stern \& Poutanen 2008), jets in a jet (Giannios et al. 2009), and plasma passing a standing shock (e.g., Marscher 2014; Zhang et al. 2014; Hervet et al. 2016; Pollack et al. 2016).

However, the details regarding the location and the mechanism of blazar emission are still not well understood (e.g., Madejski \& Sikora 2016). Simultaneous multiwavelength (MWL) observations can provide insights into the flaring mechanisms (e.g., leptonic or hadronic processes) of these objects, particularly at the wavelengths where SEDs often peak. In practice, such observations are limited in the case of fast flares at sub-hour timescales, even with dedicated strategies (e.g., Abeysekara et al. 2017). Nevertheless, contemporaneous radio data are often relevant because the radio variability timescale is usually much longer (e.g., Rani et al. 2013). In particular, the evolution of polarization (both radio and optical) before and after a gamma-ray flare provides information about magnetic field structures of the jet, and therefore the activity of possible gamma-ray-emitting regions (e.g., Zhang et al. 2014). BL Lacertae exhibits both stationary radio cores/knots and superluminal radio knots (Lister et al. 2013; Gómez et al. 2016). Possible associations between the variability of superluminal radio knots and gamma-ray flares have been investigated for BL Lacertae (e.g., Marscher et al. 2008; Arlen et al. 2013) and other blazars (e.g., Max-Moerbeck et al. 2014; Rani et al. 2014).

On 2016 October 5, we observed BL Lacertae at an elevated flux level with sub-hour variability with the Very Energetic Radiation Imaging Telescope Array System (VERITAS; see Section 2.1). A series of observations with the Very Long Baseline Array (VLBA) at $43 \mathrm{GHz}$ and $15.4 \mathrm{GHz}$ was performed over a few months before and after the gamma-ray flare, revealing a possible knot structure emerging around the time of the TeV flare (see Section 2.5). In this work, we report on the results of the VERITAS, VLBA, and other MWL observations and discuss their implications. The cosmological parameters assumed throughout this paper are $\Omega_{m}=0.27$, $\Omega_{\Lambda}=0.73$, and $H_{0}=70 \mathrm{~km} \mathrm{~s}^{-1} \mathrm{Mpc}^{-1}$ (Larson et al. 2011). At the redshift of BL Lacertae, the luminosity distance and the angular size distance are $311 \mathrm{Mpc}$ and $273 \mathrm{Mpc}$, respectively, and the angular scale is $1.3 \mathrm{pc} \mathrm{mas}^{-1}$. 


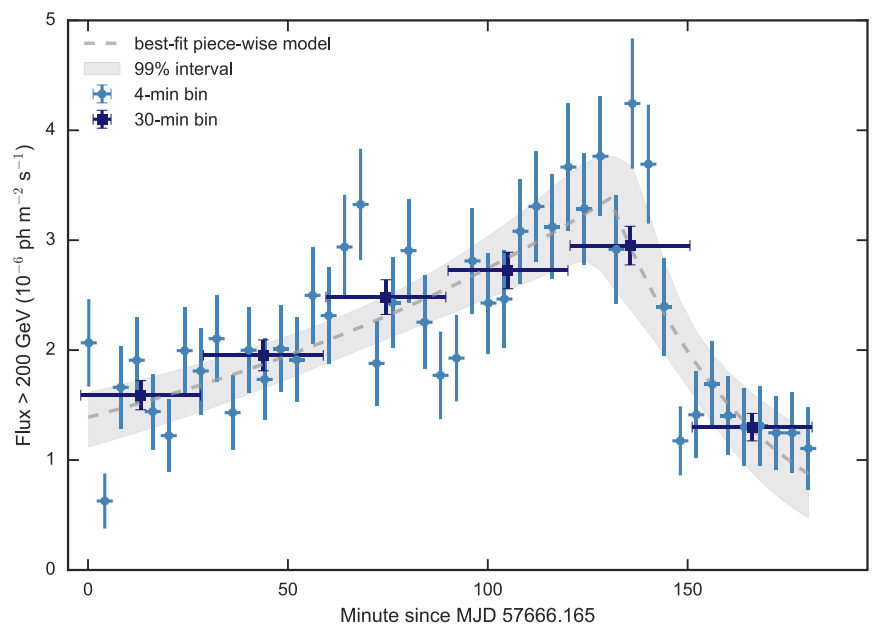

Figure 1. The VERITAS TeV gamma-ray light curves of BL Lacertae above $200 \mathrm{GeV}$ on 2016 October 5 (minute zero corresponds to 03:57:36 UTC). The light blue filled circles and the dark blue squares show the light curve in bins of 4 minutes and 30 minutes, respectively. The gray dashed line shows the model (see Equation (1)) with the best-fit parameters, and the shaded region illustrates the $99 \%$ confidence interval, both of which are derived from simulations using Markov chain Monte Carlo sampling.

\section{Observations, Data Analysis, and Results}

\subsection{VERITAS}

VERITAS is an array of four imaging atmosphericCherenkov telescopes located in southern Arizona $\left(30^{\circ} 40^{\prime} \mathrm{N}\right.$, $110^{\circ} 57^{\prime} \mathrm{W}, 1.3 \mathrm{~km}$ above sea level; Holder 2011). It is sensitive to gamma-rays in the energy range from $85 \mathrm{GeV}$ to $>30 \mathrm{TeV}$ with an energy resolution of $\sim 15 \%$ (at $1 \mathrm{TeV}$ ) and is capable of making a detection with a statistical significance of five standard deviations $(5 \sigma)$ of a point source of 0.01 C.U. in $\sim 25 \mathrm{hr}$.

BL Lacertae was observed at an elevated TeV gamma-ray flux by VERITAS on 2016 October 5 as part of an ongoing monitoring program, and follow-up observations were immediately instigated based on a real-time analysis. The total exposure of these observations amounts to 153.5 minutes after data-quality selection, with zenith angles ranging between $11^{\circ}$ and $30^{\circ}$. The data were analyzed using two independent analysis packages (Cogan 2008; Daniel 2008) and a predetermined set of cuts optimized for lower-energy showers (see e.g., Archambault et al. 2014). A detection with a statistical significance of $71 \sigma$ was made from the data on the night of the flare, with a time-averaged integral flux above $200 \mathrm{GeV}$ of $(2.24 \pm 0.06) \times 10^{-6}$ photon $\mathrm{m}^{-2} \mathrm{~s}^{-1}$ (or $\sim 0.95$ C.U.).

\subsubsection{VHE Gamma-Ray Flux Variability and the Modeling of the Flare Profile}

Figure 1 shows the VERITAS TeV gamma-ray light curve of BL Lacertae above $200 \mathrm{GeV}$ on 2016 October 5 with bins of 4 minutes and 30 minutes. A gradual rise of the TeV flux by a factor of $\sim 2$ was observed, followed by a faster decay. The measured peak flux for the 30-minute-binned light curve is $(3.0 \pm 0.2) \times 10^{-6}$ photon $\mathrm{m}^{-2} \mathrm{~s}^{-1}$, corresponding to $\sim 1.25$ C.U., and that for the 4-minute-binned light curve is $(4.2 \pm 0.6) \times 10^{-6}$ photon $\mathrm{m}^{-2} \mathrm{~s}^{-1}$, or $\sim 1.8$ C.U.

We first fitted the 4-minute-binned VERITAS light curve with a constant-flux model, obtaining a $\chi^{2}$ value of 170.8 for
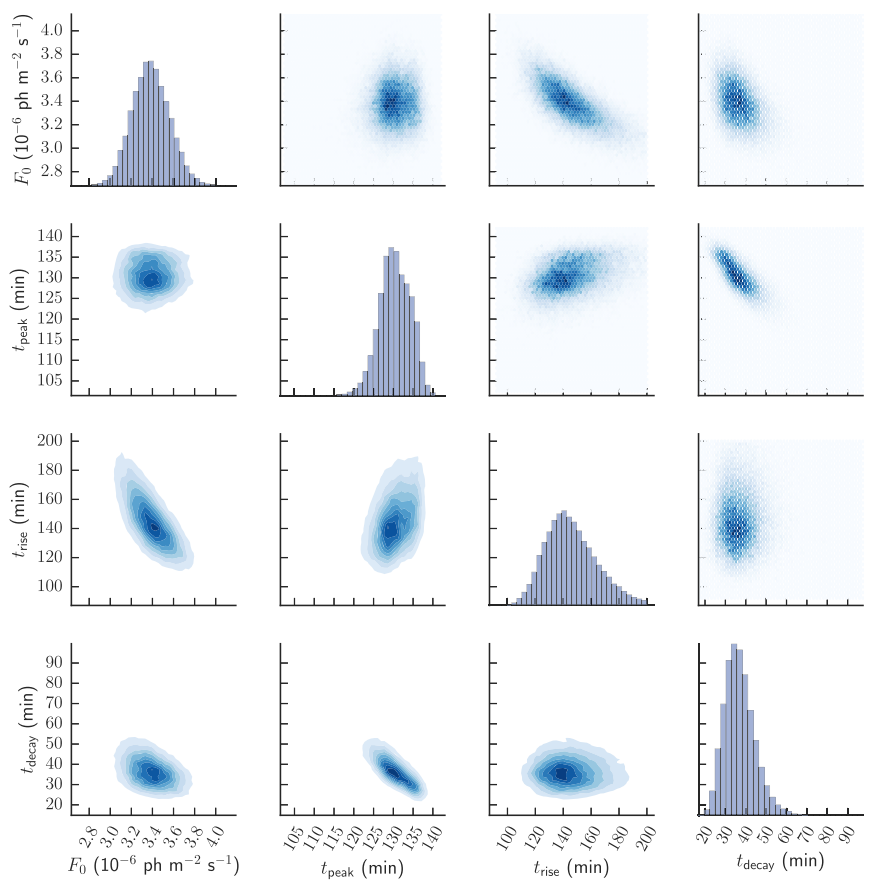

Figure 2. The joint posterior distributions of the parameters in Equation (1) obtained via MCMC simulations. The diagonal plots show the probability distribution histograms of individual parameters; the upper and lower diagonal plots show the two-dimensional histograms with hexagon binning and the kernel density estimations of the joint posterior distributions, respectively.

45 degrees of freedom (DOFs), corresponding to a $p$-value of $1.1 \times 10^{-16}$ and rejecting the constant-flux hypothesis.

To quantify the rise and decay times of the $\mathrm{TeV}$ flare, we then fitted the VHE gamma-ray light curve with a piecewise exponential function as follows:

$$
F(t)= \begin{cases}F_{0} e^{\left(t-t_{\text {peak }}\right) / t_{\text {rise }}}, & t \leqslant t_{\text {peak }} \\ F_{0} e^{-\left(t-t_{\text {peak }}\right) / t_{\text {decay }}}, & t>t_{\text {peak }}\end{cases}
$$

where $F_{0}$ is the peak flux, $t_{\text {peak }}$ is the time of the peak flux, and $t_{\text {rise }}$ and $t_{\text {decay }}$ are the rise and decay times, respectively, on which the flux varies by a factor of $e$.

The optimal values of the parameters and their uncertainties were determined from the posterior distributions obtained from Markov chain Monte Carlo (MCMC) simulations, for which the Python package emcee (Foreman-Mackey et al. 2013) was used. The MCMC chain contains 100 random walkers in the parameter space initialized with a uniform random prior. Each random walker walks 4000 steps, the first 2000 steps of which are discarded as the "burn-in" samples. This amounts to $2 \times 10^{5}$ effective MCMC simulations. A proposal scale parameter was chosen so that the mean proposal acceptance fraction is $37 \%$, ensuring an adequate yet efficient sampling of the posterior distributions. Note that the parameters are bounded to be positive, so that they are physically meaningful, and sufficiently large upper bounds were also provided for computational efficiency. After the posterior distributions were obtained, kernel density estimation with Gaussian kernels of bandwidths equal to $1 \%$ of the range of the corresponding parameter was used to estimate the most likely value (maximum a posteriori) and the $68 \%$ confidence interval of each parameter.

The joint posterior distributions of the parameters from the MCMC sampling are shown in Figure 2. The diagonal plots 
show the posterior probability distributions of each parameter, some of which (e.g., $t_{\text {peak }}$ ) appear non-Gaussian. Correlations

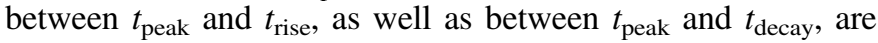
also apparent in the off-diagonal joint distributions. The best-fit model and the $99 \%$ confidence intervals from the MCMC sampling are shown in Figure 1. The rise and decay times of the flare are determined to be $140_{-11}^{+25}$ minutes and $36_{-7}^{+8}$ minutes, respectively. The best-fit peak time and flux are $130_{-3}^{+5}$ minutes (after MJD 57666.165) and 3.4 $4_{-0.2}^{+0.2} \times 10^{-6}$ photon $\mathrm{m}^{-2} \mathrm{~s}^{-1}$, respectively.

Further VERITAS observations of BL Lacertae were made on 2016 October 6 with 37.6 minutes live exposure, and from October 22 to November 19 with 294.6 minutes live exposure, after data-quality selection; neither of these sets of observations led to a detection of the source (signal significances of only $2.6 \sigma$ and $0.9 \sigma$, respectively). The upper limits on integral flux (shown in the first panel of Figure 5) between 0.2 and $30 \mathrm{TeV}$ at $99 \%$ confidence level from the observations on October 6 and between October 22 and November 19 were obtained as $2.0 \times 10^{-7}$ photon $\mathrm{m}^{-2} \mathrm{~s}^{-1}$ and $2.8 \times 10^{-8}$ photon $\mathrm{m}^{-2} \mathrm{~s}^{-1}$, respectively, assuming a power-law spectrum with a photon index of 3.3 (see Section 2.1.2).

Motivated by the existence of multiple radio emission zones identified in VLBA data (see Section 2.5) and several multizone models for BL Lacertae that are consistent with past observations (e.g., Raiteri et al. 2013; Hervet et al. 2016), we also fitted the light curve with a model including an additional constant-flux baseline. In a multi-zone model, different zones can be of different sizes and vary independently on different timescales. Therefore, it is possible to have a larger emitting zone that varies slowly, which can be adequately described by a constant-baseline component on the timescale considered, and a smaller, more energetic zone that is responsible for the rapid flare described by the exponential components. With the more complex model, the best-fit decay time is only $2.6_{-0.8}^{+6.7}$ minutes, with a baseline flux of $1.2_{-0.2}^{+0.1} \times 10^{-6}$ photon $\mathrm{m}^{-2} \mathrm{~s}^{-1}$. This best-fit baseline flux is higher than the upper limit obtained from the observations on the next day, indicating that the slower component would be required to vary on timescales of $\sim 1$ day, consistent with the GeV gamma-ray observations (Section 2.2). However, we would like to highlight that it is not possible to unambiguously reject either model based on the statistics.

\subsubsection{The VHE Spectrum}

A power-law fit to the VERITAS spectrum of BL Lacertae yields a best-fit photon index of 3.28 and a reduced $\chi^{2}$ value $\chi^{2} / \mathrm{DOF}=30.6$, indicating that a simple power law does not adequately describe the spectrum.

A log-parabola model fits the VERITAS spectrum better:

$$
\begin{aligned}
\frac{d N}{d E}= & (2.22 \pm 0.07) \times 10^{-5} \\
& \times\left(\frac{E}{0.2 \mathrm{TeV}}\right)^{\left[-(2.4 \pm 0.1)-(1.8 \pm 0.3) \log _{10}\left(\frac{E}{0.2 \mathrm{TeV}}\right)\right]} \\
& \times \mathrm{m}^{-2} \mathrm{~s}^{-1} \mathrm{TeV}^{-1},
\end{aligned}
$$

with $\chi^{2} / \mathrm{DOF}=1.6$.

After de-absorbing the VHE spectrum using the optical depths for a source at a redshift of 0.069 according to the model of extragalactic background light in Domínguez et al. (2011),

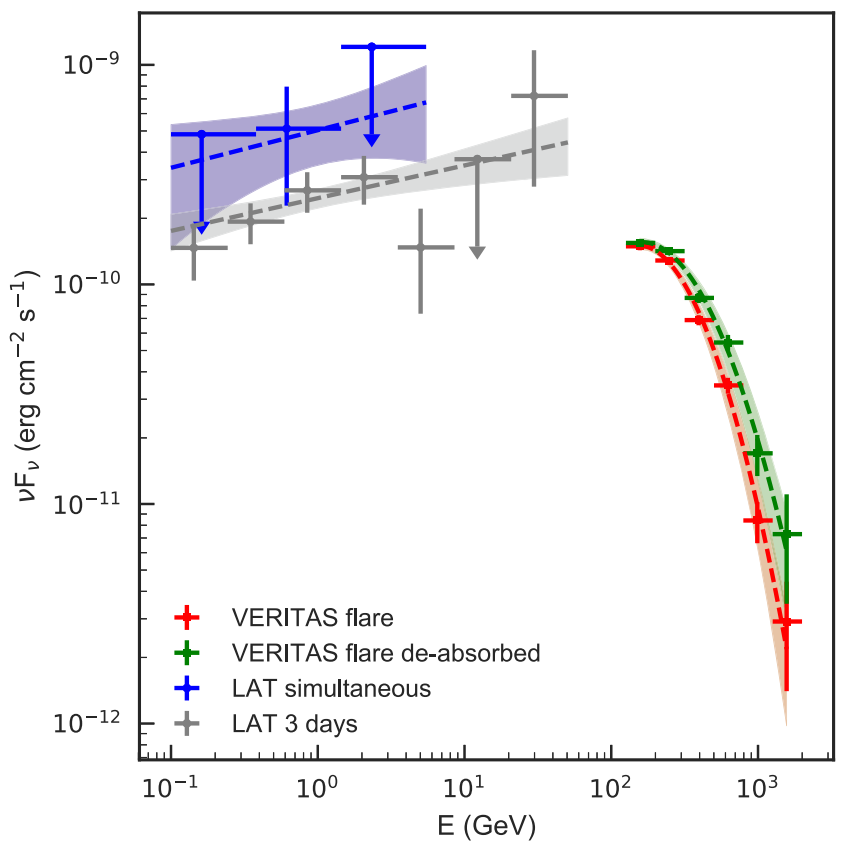

Figure 3. The gamma-ray SEDs of BL Lacertae measured by Fermi-LAT and VERITAS. The Fermi-LAT SEDs that are strictly simultaneous with VERITAS observations on the night of the flare (2016 October 5) and from the three days around it are shown in blue and gray, respectively. The observed and de-absorbed VERITAS SEDs averaged over all observations on the night of the flare are shown in red and green, respectively. Each shaded region is derived from the $1 \sigma$ confidence intervals of the best-fit parameters for the corresponding spectrum.

the best-fit log-parabola model becomes

$$
\begin{aligned}
\frac{d N}{d E}= & (2.36 \pm 0.07) \times 10^{-5} \\
& \times\left(\frac{E}{0.2 \mathrm{TeV}}\right)^{\left[-(2.2 \pm 0.1)-(1.4 \pm 0.3) \log _{10}\left(\frac{E}{0.2 \mathrm{TeV}}\right)\right]} \\
& \times \mathrm{m}^{-2} \mathrm{~s}^{-1} \mathrm{TeV}^{-1},
\end{aligned}
$$

with $\chi^{2} / \mathrm{DOF}=1.7$. The observed and de-absorbed $\mathrm{TeV}$ gamma-ray spectra are shown together with the GeV gammaray spectra (Section 2.2) in Figure 3 in the $\nu F_{\nu}$ representation.

\subsection{Fermi-LAT}

The Large Area Telescope (LAT) on board the Fermi satellite is a pair-conversion gamma-ray telescope sensitive to energies from $\sim 20 \mathrm{MeV}$ to $>300 \mathrm{GeV}$ (Atwood et al. 2009).

An unbinned likelihood analysis was performed with the LAT ScienceTools v10r0p5 and Pass-8 P8R2_SOURCE_V6_v06 instrument response functions (Atwood et al. 2013). SOURCE class events with energy between $100 \mathrm{MeV}$ and $300 \mathrm{GeV}$ within $10^{\circ}$ of the position of BL Lacertae were selected. For the short durations of interest to the $\mathrm{TeV}$ flare, a simple model containing BL Lacertae, another point source 3FGL J2151.6+4154 2 away from BL Lacertae, and the contributions from the Galactic (gl1_iem_v06) and isotropic (iso_P8R2_SOURCE_V6_V06) diffuse emission were included. A maximum zenith angle cut of $90^{\circ}$ was applied. We checked in the residual test-statistics map that no significant excess was left unaccounted for within the model. For the short durations, a power law was used to model BL Lacertae instead of the log-parabola model used in the 3FGL 
catalog. We verified with an analysis using a log-parabola spectral model and obtained consistent flux values.

For the light curve shown in the second panel of Figure 5, an unbinned likelihood analysis was performed on each one-day interval, leaving the normalizations and power-law indices of BL Lacertae and 3FGL J2151.6+4154 free, as well as the normalization of the diffuse components. The source was in an elevated $\mathrm{GeV}$ gamma-ray state when the $\mathrm{TeV}$ flare was observed, although the $\mathrm{GeV}$ flux varied on a much longer timescale. An exponential fit to a 15 day interval around the $\mathrm{TeV}$ gamma-ray flare yields a rise time of $2.1 \pm 0.2$ days and a decay time of $7 \pm 2$ days.

The gamma-ray SEDs measured by the Fermi-LAT and VERITAS on the night of the TeV flare are shown in Figure 3. In order to obtain the GeV gamma-ray SEDs, we used the usercontributed tool likeSED.py ${ }^{40}$ to perform the unbinned likelihood analysis in several energy bands. The power-law index that gives the best fit to the LAT data completely simultaneous with VERITAS is $1.83 \pm 0.21$, which is similar to that of the three-day binned LAT data, $1.85 \pm 0.07$. These values indicate a harder $\mathrm{GeV}$ gamma-ray spectrum during the flare than that reported in the 3FGL catalog (with an index of 2.25; Acero et al. 2015).

Both the $\mathrm{GeV}$ and $\mathrm{TeV}$ gamma-ray spectral indices of this flare in 2016 are comparable to those of the flare in 2011 (Arlen et al. 2013), and they suggest that the peak energy of the gamma-ray SED during the flare is between $5 \mathrm{GeV}$ and $100 \mathrm{GeV}$.

\subsection{Swift-XRT}

The X-Ray Telescope (XRT) on board the Swift satellite is a grazing-incidence focusing X-ray telescope, and is sensitive to photons in the energy range $0.2-10 \mathrm{keV}$ (Gehrels et al. 2004; Burrows et al. 2005).

Follow-up observations of BL Lacertae were carried out with the Swift-XRT on 2016 October 6, 7, and 8; the only other XRT observations within a 45 day window around the time of the VHE flare were made on 2016 October 27 and November 2. The XRT data, taken in the photon-counting (PC) mode, were analyzed using the HEAsoft package (v6.19). The data were first processed using xrtpipeline (v0.13.2) with calibration database (CALDB v20160706). The count rates in the PC mode were $>0.5$ count $\mathrm{s}^{-1}$, and the effect of potential pile-up was checked for all observations by fitting a King function to the point-spread functions at $>15$ arcsec (Moretti et al. 2005). Those central pixels where the data fall below the model curve, indicating pile-up, were excluded.

For the observations on 2016 October 6, the King function agrees with the data even on the brightest pixels. Therefore, a circular source region of a radius of 20 pixels centered on BL Lacertae was used. Annular source regions were used for the data taken on 2016 October 7 and 8, with inner radii of four and two pixels, and an outer radius of 20 pixels. For all three observations, an annular background region with inner and outer radii of 70 and 120 pixels, respectively, was used. Note that source regions excluding the central two and four pixels were also tested for the observations on 2016 October 6 , and consistent results were obtained. Therefore, we are confident that no bias was introduced by the different exclusion regions used for pile-up correction.

\footnotetext{
${ }^{40}$ https://fermi.gsfc.nasa.gov/ssc/data/analysis/user/
}

Table 1

Swift-XRT Spectral-fit Results Using the Absorbed-power-law Model Described in Equation (4), with $N_{H}$ Free and Fixed

\begin{tabular}{lccrc}
\hline \hline Date & $\alpha$ & $\begin{array}{c}K \\
\left(10^{-2} \mathrm{keV}^{-1} \mathrm{~cm}^{-2} \mathrm{~s}^{-1}\right)\end{array}$ & $\begin{array}{c}N_{H} \\
\left(10^{21} \mathrm{~cm}^{-2}\right)\end{array}$ & $\chi^{2} / \mathrm{DOF}$ \\
\hline Oct 6 & $2.5 \pm 0.1$ & $0.62_{-0.06}^{+0.07}$ & $2.7_{-0.3}^{+0.3}$ & 0.83 \\
Oct 7 & $2.1 \pm 0.1$ & $4.6_{-0.5}^{+0.6}$ & $3.1_{-0.4}^{+0.5}$ & 1.07 \\
Oct 8 & $2.3 \pm 0.1$ & $0.43_{-0.05}^{+0.06}$ & $3.0_{-0.4}^{+0.5}$ & 0.54 \\
Oct 27 & $1.4_{-0.3}^{+0.4}$ & $0.14_{-0.04}^{+0.08}$ & $1.2_{-1.2}^{+2.2}$ & 0.48 \\
Nov 2 & $1.3 \pm 0.3$ & $0.11_{-0.03}^{+0.04}$ & $1.4_{-0.8}^{+1.1}$ & 0.33 \\
\hline Oct 6 & $2.54 \pm 0.07$ & $0.65 \pm 0.03$ & 2.9 (fixed) & 0.82 \\
Oct 7 & $2.08 \pm 0.07$ & $4.34 \pm 0.24$ & 2.9 (fixed) & 1.04 \\
Oct 8 & $2.26 \pm 0.08$ & $0.41 \pm 0.02$ & 2.9 (fixed) & 0.52 \\
Oct 27 & $1.64 \pm 0.20$ & $0.19 \pm 0.03$ & 2.9 (fixed) & 0.50 \\
Nov 2 & $1.76 \pm 0.19$ & $0.17 \pm 0.02$ & 2.9 (fixed) & 0.63 \\
\hline
\end{tabular}

Note. The errors quoted denote $68 \%$ confidence intervals.

The observations on October 7 consisted of two intervals of duration $486 \mathrm{~s}$ and $1422 \mathrm{~s}$, separated by roughly one satellite orbital period ( $\sim 90$ minutes). A sustained dark stripe (likely due to bad CCD columns) appears in the XRT image near the position of BL Lacertae, contaminating the second interval. Therefore, we conservatively chose to use only the data recorded during the first interval. The image and spectrum of each $\sim 3$ minutes of this relatively short exposure were checked for data quality, and no anomaly was found.

Ancillary response files were generated using the xrtmkarf task with the response matrix file swxpc0to12s6_ 20130101v014.rmf. The spectrum was fitted with an absorbed-power-law model (po*wabs):

$$
\frac{d N}{d E}=e^{-N_{H} \sigma(E)} K\left(\frac{E}{1 \mathrm{keV}}\right)^{-\alpha},
$$

where $N_{H}$ is the column density of neutral hydrogen, $\sigma(E)$ is the photoelectric cross section, and $K$ and $\alpha$ are the normalization and index of the power-law component, respectively. The bestfit values of the parameters are shown in Table 1. Note that the best-fit values of $N_{H}$ are in agreement with the archival results from X-ray spectral fit (Madejski et al. 1999; Ravasio et al. 2003; Arlen et al. 2013), but are larger than the value $N_{H}=1.8 \times 10^{21} \mathrm{~cm}^{-2}$ from the Leiden/Argentine/Bonn (LAB) survey of Galactic H I (Kalberla et al. 2005). This difference is likely due to the additional contribution of the Galactic molecular gas (e.g., CO emission), because BL Lacertae is relatively close to the Galactic plane (with a Galactic latitude $b=-10^{\circ} .44$ ) (Madejski et al. 1999). As the $N_{H}$ value is expected to stay constant over the period of interest, we also fit the same model with $N_{H}$ fixed at the average best-fit value $N_{H}=2.9 \times 10^{21} \mathrm{~cm}^{-2}$ over the three nights of XRT observations, to better constrain the spectral index and normalization. We also investigated an absorbed-log-parabola model with $N_{H}$ fixed at $2.9 \times 10^{21} \mathrm{~cm}^{-2}$ to fit the X-ray spectra. We see no evidence for spectral curvature, as the bestfit log-parabola model reduces to a power law. The X-ray SEDs of BL Lacertae measured on 2016 October 6, 7, and 8 are shown in Figure 4. The X-ray emission from the source was stronger and harder on 2016 October 7 (two days after the TeV gamma-ray flare) compared to the day before and the day after 

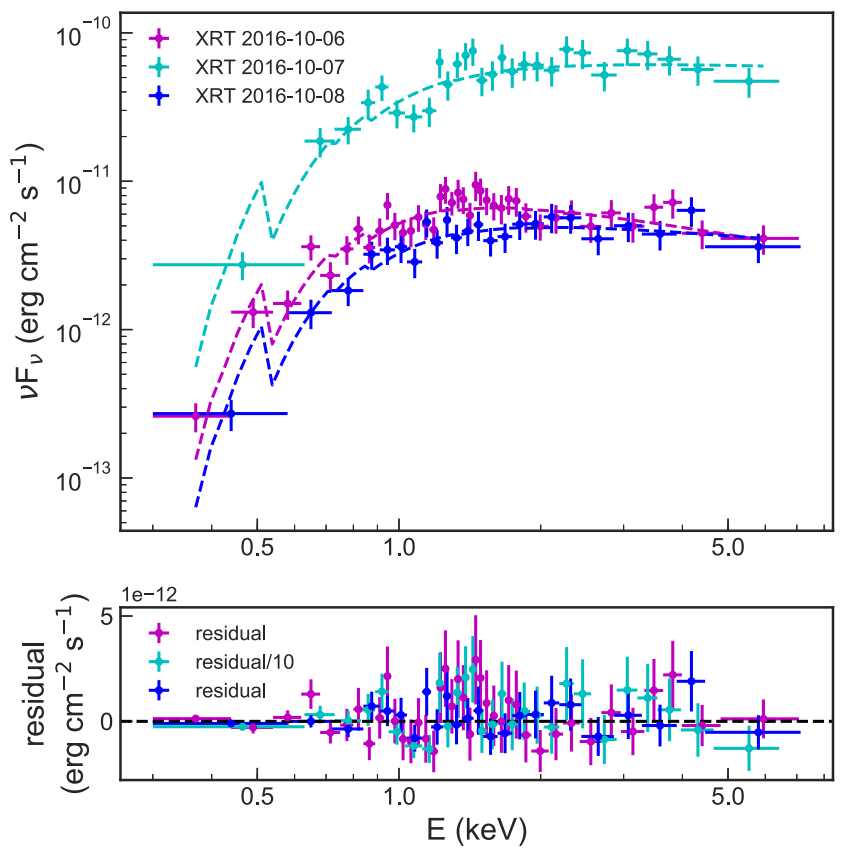

Figure 4. Top: the X-ray SEDs measured by Swift-XRT on 2016 October 6, 7, and 8 . The dashed lines are the best-fit absorbed-power-law model with $N_{H}$ fixed at $2.9 \times 10^{21} \mathrm{~cm}^{-2}$. Bottom: the distributions of the fit residuals of each X-ray SED. Note that the residual values on October 7, shown in cyan, are divided by 10 to facilitate comparison with the other two distributions shown.

(see Table 1). The energy flux values based on the best-fit absorbed-power-law model between $0.3 \mathrm{keV}$ and $10 \mathrm{keV}$ on 2016 October 6,7 , and 8 were $(1.4 \pm 0.1),(14.2 \pm 0.9)$, and $(1.1 \pm 0.1) \times 10^{-11} \mathrm{erg} \mathrm{cm}^{-2} \mathrm{~s}^{-1}$, respectively, as shown in the third panel of Figure 5 along with the results from two more observations taken on 2016 October 27 and November 2.

\subsection{Optical Facilities}

BL Lacertae was monitored in the $R$-band at high cadence by a number of optical facilities, including the Steward Observatory $^{41}$ (Smith et al. 2009), the AZT-8 reflector of the Crimean Astrophysical Observatory, the Perkins telescope, ${ }^{42}$ the LX-200 telescope in St. Petersburg, Russia, and the Calar Alto $2.2 \mathrm{~m}$ Telescope (with observations obtained through the MAPCAT $^{43}$ program) in Almería, Spain. We also included the $r^{\prime}$-band observations made with the 48 inch telescope at the Fred Lawrence Whipple Observatory (FLWO). We transformed the $r^{\prime}$-band flux to $R$-band using the color index $V-R=0.73 \pm 0.19$ for BL Lacertae (Fan et al. 1998) and the transformation $r^{\prime}-R=0.19(V-R)+0.13$ (Smith et al. 2002). The dominant uncertainty from the conversion to $R$-band comes from the variability of the $V-R$ color index, resulting in an additional systematic uncertainty of $\sim 0.04$ magnitude, which is included in the converted FLWO $R$-band magnitude shown in Figure 5. Any variability in the color index $\Delta(V-R)<0.19$ during the epoch of observations shown would lead to a shift of the FLWO $R$-band magnitude within the error bars shown.

\footnotetext{
41 http://james.as.arizona.edu/ psmith/Fermi

42 http://www.lowell.edu/research/research-facilities/1-8-meter-perkins/

43 MAPCAT stands for the Monitoring of AGN with Polarimetry at the Calar Alto Telescopes, see: http://www.iaa.es/ iagudo/_iagudo/MAPCAT.html.
}

The $R$-band flux and polarization measurements contemporaneous with the gamma-ray flare are shown in Figure 5. The lower three panels (from top to bottom) show the $R$-band magnitude, polarization fraction, and electric vector position angle (EVPA) of the source, respectively. A $-180^{\circ}$ shift is applied to all the EVPA measurements before MJD 57662, so that the EVPA difference between MJD 57662 and 57658 is reduced to $\sim 80^{\circ}$ from $\sim 100^{\circ}$ before the shift was applied (see, e.g., Abdo et al. 2010). The measurements are reasonably consistent between the various instruments.

The $R$-band flux from the source varied in a similar manner to the GeV flux, with an increase observed a few days before the VHE flare. The optical EVPA appeared to have rotated smoothly from roughly perpendicular to the position angle (PA) of the jet in late 2016 September to roughly parallel in late 2016 October, except for three days before the TeV gamma-ray flare when the optical EVPA was nearly aligned with the PA of the jet. This was followed by a sudden decrease in EVPA on the day before the $\mathrm{TeV}$ gamma-ray flare. The fractional polarization was relatively low around the time of the $\mathrm{TeV}$ flare, and increased to the highest value of the 45 day period in late October when the EVPA was again aligned with the jet.

\subsection{Radio Facilities}

BL Lacertae was observed throughout the period of interest at $43 \mathrm{GHz}$ with the VLBA under the VLBA-BU-Blazar monitoring program (Jorstad \& Marscher 2016) and at 15.4 GHz with the Monitoring Of Jets in AGNs with VLBA Experiments (MOJAVE) program (Lister et al. 2009). The $43 \mathrm{GHz}$ and $15.4 \mathrm{GHz}$ VLBA data calibration and imaging procedures were identical to those described by Jorstad et al. (2005) and Lister et al. (2009), respectively.

Figure 6 presents $43 \mathrm{GHz}$ images of the parsec-scale jet of BL Lacertae at five epochs from 2016 September 5 to December 23. The second epoch, 2016 October 6, took place only one day after the VHE flare. The images are convolved with a circular Gaussian restoring beam with a FWHM of 0.1 mas, which is similar to the angular resolution of the longest baselines along the (southern) direction of the jet. We note that the October 6 observation was affected by equipment failure at the Maunakea and Hancock antennas, at the extremities of the array, although this degraded the north-south angular resolution by only $14 \%$. The corresponding linear resolution at the redshift of BL Lacertae is $0.13 \mathrm{pc}$ in projection on the sky and $1.8_{-0.4}^{+0.8} \mathrm{pc}$ if we adopt a viewing angle of $4.2 \pm 1.3$ between the jet axis and line of sight (Jorstad et al. 2017).

As was the case in previous observations (Jorstad et al. 2005; Arlen et al. 2013; Gómez et al. 2016; Wehrle et al. 2016), the main structure of the jet consists of three quasi-stationary brightness peaks, designated as $A O, A l 0.12$ mas to the south of $A 0$, and $A 20.30$ mas to the south of $A 0$. The locations of $A 1$ and $A 2$ appear to fluctuate as moving emission features (frequently referred to as "knots") with superluminal apparent velocities pass through the region. Such a combination of moving and stationary emission components complicates the interpretation of the changing structures of the total and polarized intensities. Because of this, the interpretation that we offer to explain the variations within the images is not unique.

We ignore the effects of Faraday rotation on the polarization EVPA, which Jorstad et al. (2007) estimated to be low $\left(-16^{\circ}\right)$ between $43 \mathrm{GHz}$ and $300 \mathrm{GHz}$. It is worth mentioning that Hovatta et al. (2012) measured a much lower (by an order of 


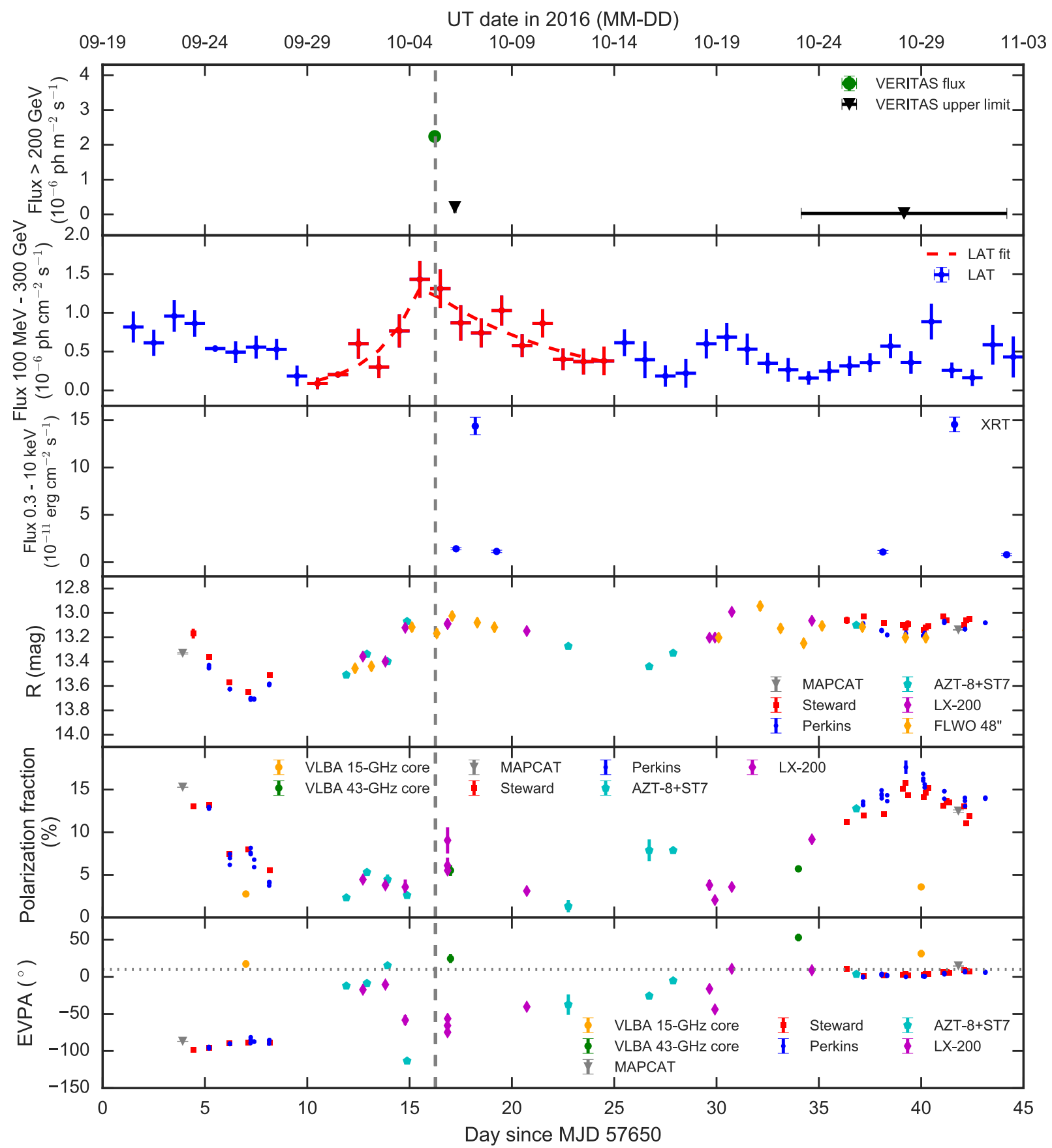

Figure 5. The 45 day MWL light curves of BL Lacertae around the time of the VHE flare. The top panel shows the TeV gamma-ray flux measured by VERITAS on the night of the flare, as well as the upper limits obtained later. The second panel shows the daily-binned GeV gamma-ray light curve measured by Fermi-LAT, as well as a piecewise exponential fit (see Equation (1)) to a 15 day interval around the TeV gamma-ray flare (red dashed line). The third panel shows the X-ray energy flux from the five Swift-XRT observations. The bottom three panels show the $R$-band photometric and polarimetric measurements (see Section 2.4). The gray vertical dashed line shows the peak time of the TeV gamma-ray flare observed by VERITAS. The fractional polarizations and the EVPAs of the $43 \mathrm{GHz}$ and $15 \mathrm{GHz}$ core are also shown in the bottom two panels. The gray horizontal dotted line in the bottom panel shows the position angle of the jet.

magnitude) Faraday rotation using $8-15 \mathrm{GHz}$ observations. This could be due to a combination of a possible variability in the rotation measure and a decrease of the rotation measure with distance from the central black hole (Jorstad et al. 2007), because the core at $15 \mathrm{GHz}$ is located further away from the black hole than the core at $43 \mathrm{GHz}$ due to the effect of opacity.

A knot of emission with enhanced polarization at $43 \mathrm{GHz}$, which we designate as $K 16$, appears to propagate down the jet. Its centroid moves from $\sim 0.05$ mas south of $A O$ on October 23 to $\sim 0.28$ mas from $A O$ on December 23. This corresponds to an apparent speed of $6 c$, within the range typically observed in BL Lacertae (Jorstad et al. 2005, 2017; Marscher et al. 2008; Arlen et al. 2013; Lister et al. 2013; Wehrle et al. 2016). Extrapolation back to October 6 places the knot K16 0.01 mas north of the centroid of $A 0$, within the $A O$ emission region characterized by its angular size of $0.03 \pm 0.02$ mas (Jorstad et al. 2017). This implies that the VHE flare occurred as the moving knot crossed the stationary "core," which Marscher et al. (2008) have interpreted as a standing shock located $\sim 1 \mathrm{pc}$ from the central black hole. 


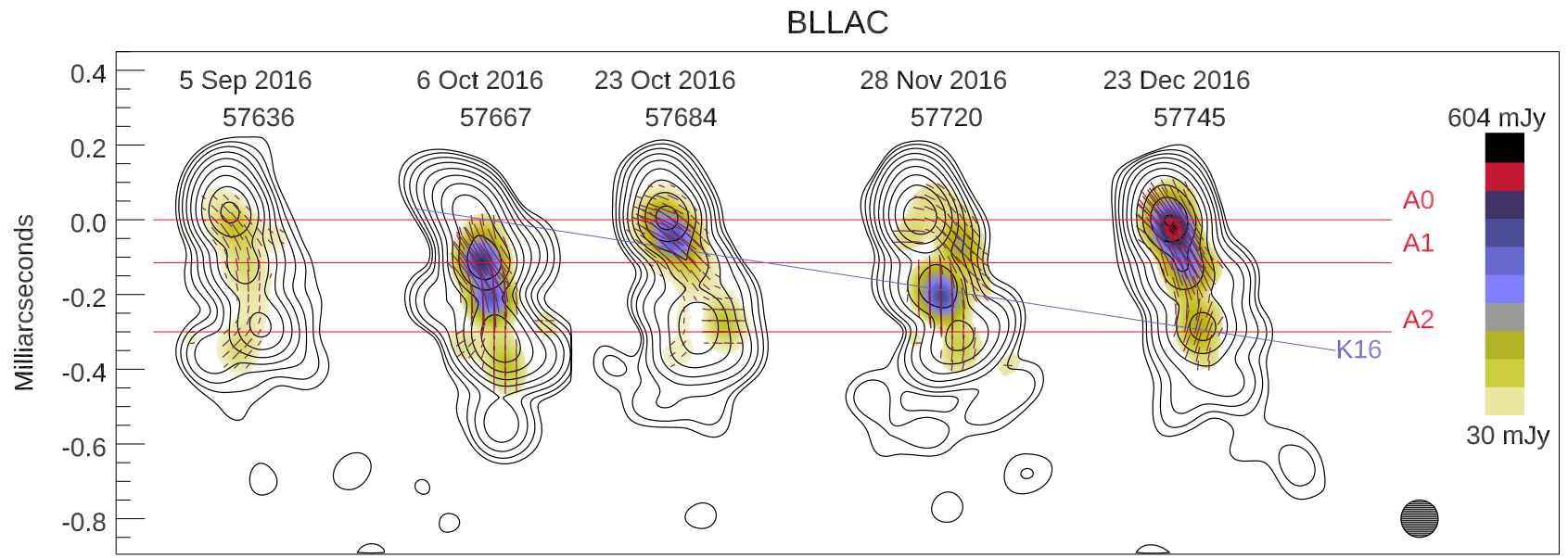

Figure 6. The $43 \mathrm{GHz}$ VLBA total (contours) and polarized (color scale) intensity images of BL Lac. The total intensity peak is $1.15 \mathrm{Jy}^{\mathrm{J}}$ beam ${ }^{-1}$. The contours are 0.2 , $0.4,0.8, \ldots, 51.2,96 \%$ of the peak. The restoring beam shown in the bottom right corner is a circular Gaussian with FWHM $=0.1$ mas. Linear segments within the images indicate position angles of the polarization, with the length of segments proportional to the local polarized intensity. Red horizontal lines mark the mean locations of the three quasi-stationary features; the blue line across the epochs from 2016 October to December traces the motion of the superluminal knot K16.

The VLBA images at $15.4 \mathrm{GHz}$, as shown in Figure 7, reveal the evolution of the jet structures further away from the central source and on a larger spatial scale, as a result of optical depth and angular resolution, respectively, compared with the observations at $43 \mathrm{GHz}$. Therefore, a delay is expected between the measurements at these two frequencies. The polarized intensity of the stationary core of BL Lacertae at $15.4 \mathrm{GHz}$ reached a minimum on 2016 December 26 and gradually increased, with a potentially bright feature with distinct polarization angle (consistent with the EVPAs measured at $43 \mathrm{GHz}$ on 2016 December 23), which may correspond to the knot $K 16$ observed at $43 \mathrm{GHz}$ earlier, appearing at $\sim 1$ mas southwest of the core. This is consistent with past observations of the same source with the VLBA at different frequencies reported by Bach et al. (2006), where new components of the jet were seen to fade as they separated from the core, disappearing at $\sim 0.7$ mas and reappearing at $\sim 1$ mas.

We show the fractional polarizations and EVPAs of the $43 \mathrm{GHz}$ and $15 \mathrm{GHz}$ core in the bottom two panels of Figure 5, along with the $R$-band results. The EVPAs of the core at $43 \mathrm{GHz}$ and $15 \mathrm{GHz}$ are roughly consistent with the PA of $10^{\circ}$ of the jet over the course of a few months since 2016 September. This implies that the magnetic field is toroidal/ helical at the core, as we discuss in Section 3. Further downstream in the jet, the EVPAs become more perpendicular to the PA of the jet, as shown in Figure 7. Such locationdependent radio EVPAs help us to interpret the dominant optical component based on the optical EVPA data.

We show in Figure 8 the evolution of the total flux density of BL Lacertae, measured by the Metsähovi Radio Observatory at $37 \mathrm{GHz}$ and by Owens Valley Radio Observatory (OVRO) at $15 \mathrm{GHz}$, over about a year, as well as their $z$-transformed discrete cross-correlation (ZDCF; Alexander 2013).

The $37 \mathrm{GHz}$ observations were made with the $13.7 \mathrm{~m}$ diameter Aalto University Metsähovi radio telescope, which is a radome-enclosed Cassegrain-type antenna situated in Finland. The measurements were made with a dual-beam receiver of $1 \mathrm{GHz}$ bandwidth centered at $36.8 \mathrm{GHz}$. The front end, a pseudomorphic transistor of high electron mobility, operates at room temperature. The $37 \mathrm{GHz}$ observations are Dicke-switched ON-ON observations, alternating the source and the sky in each feed horn to remove atmospheric and ground contamination. The typical integration time to obtain one flux-density data point is between 1200 and $1600 \mathrm{~s}$. The detection limit of the telescope at $37 \mathrm{GHz}$ is of the order of 0.2 Jy under optimal conditions. Data points with a signal-tonoise ratio $<4$ are handled as non-detections.

The flux-density scale is set by observations of the $\mathrm{H}$ II region of DR 21, while NGC 7027, 3C 274, and 3C 84 are used as secondary calibrators. A detailed description of the data reduction and analysis can be found in Teräsranta et al. (1998). The error estimate in the flux density includes the contributions from the measurement rms and the uncertainty of the absolute calibration.

The OVRO $40 \mathrm{~m}$ telescope uses off-axis dual-beam optics and a cryogenic pseudo-correlation receiver with a $15.0 \mathrm{GHz}$ center frequency and $3 \mathrm{GHz}$ bandwidth. The source is alternated between the two beams in an $\mathrm{ON}-\mathrm{ON}$ fashion to remove atmospheric and ground contamination. The fast gain variations are corrected using a $180^{\circ}$ phase switch. Calibration is achieved using a temperature-stable diode noise source to compensate for drifts in receiver gain, and the flux-density scale is derived from observations of 3C 286 assuming the value of $3.44 \mathrm{Jy}$ at $15.0 \mathrm{GHz}$ reported by Baars et al. (1977). The systematic uncertainty in the flux-density scale is $\sim 5 \%$, which is not included in the error bars in Figure 8. Complete details of the reduction and calibration procedures are given in Richards et al. (2011).

The $37 \mathrm{GHz}$ and $15 \mathrm{GHz}$ light curves show that at the time of the $\mathrm{TeV}$ gamma-ray flare, BL Lacertae was transitioning from a steady radio flux state to a flaring state that lasted for about five months. The ZDCF shows no significant detection of any time lag between the fluxes at the two frequencies, suggesting that both observations are dominated by the flux from a region that is optically thin at $15 \mathrm{GHz}$.

\section{Discussion}

For the second time, VERITAS has detected a fast gammaray flare from BL Lacertae.

While no information was obtained from the rising phase of the first VHE flare in 2011 (Arlen et al. 2013), the VERITAS measurements during the 2016 flare described in this work cover both the rise and decay phases of the flare. 

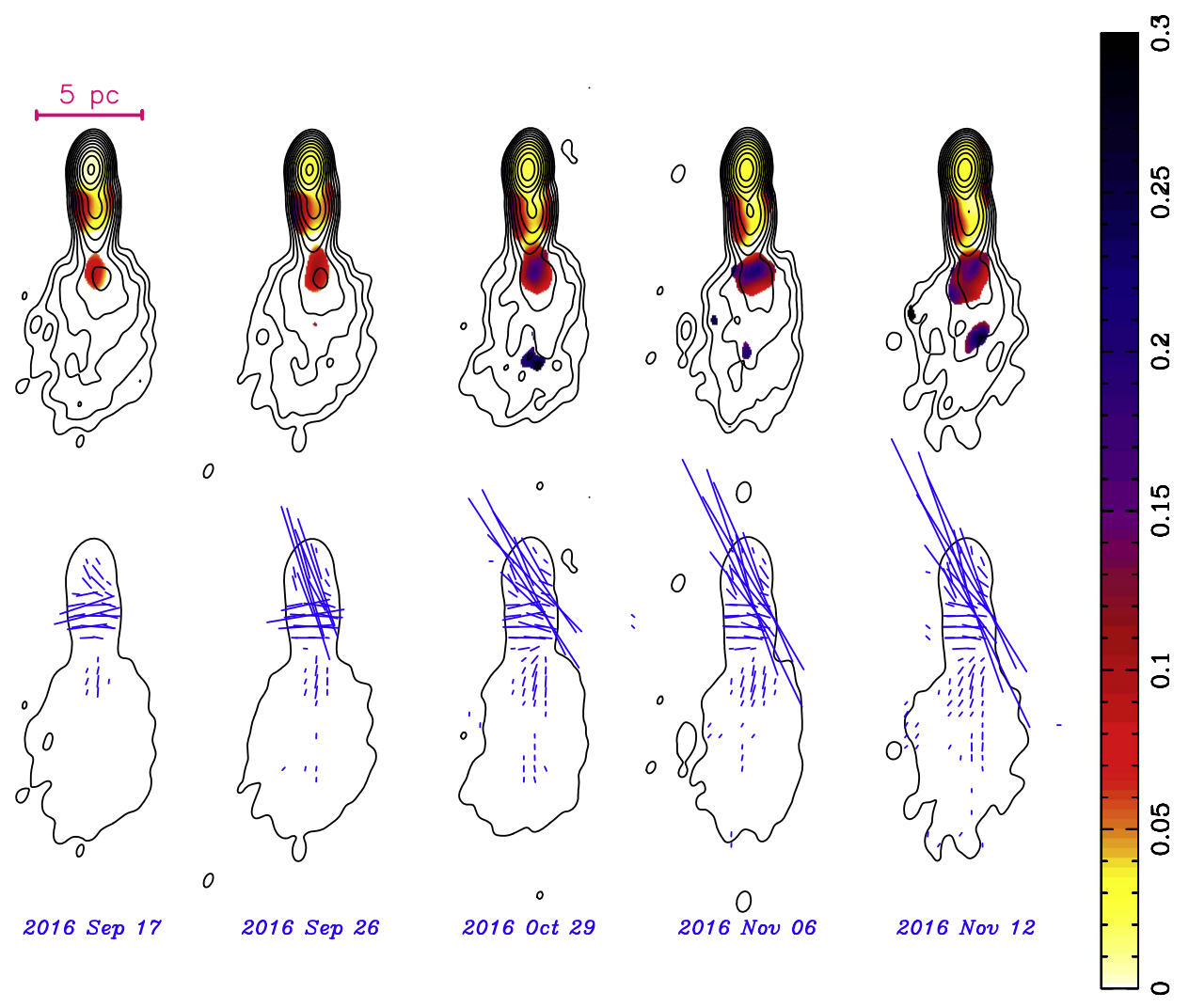

$2016 \operatorname{Sep} 26$

2016 Oct 29

2016 Nov 06

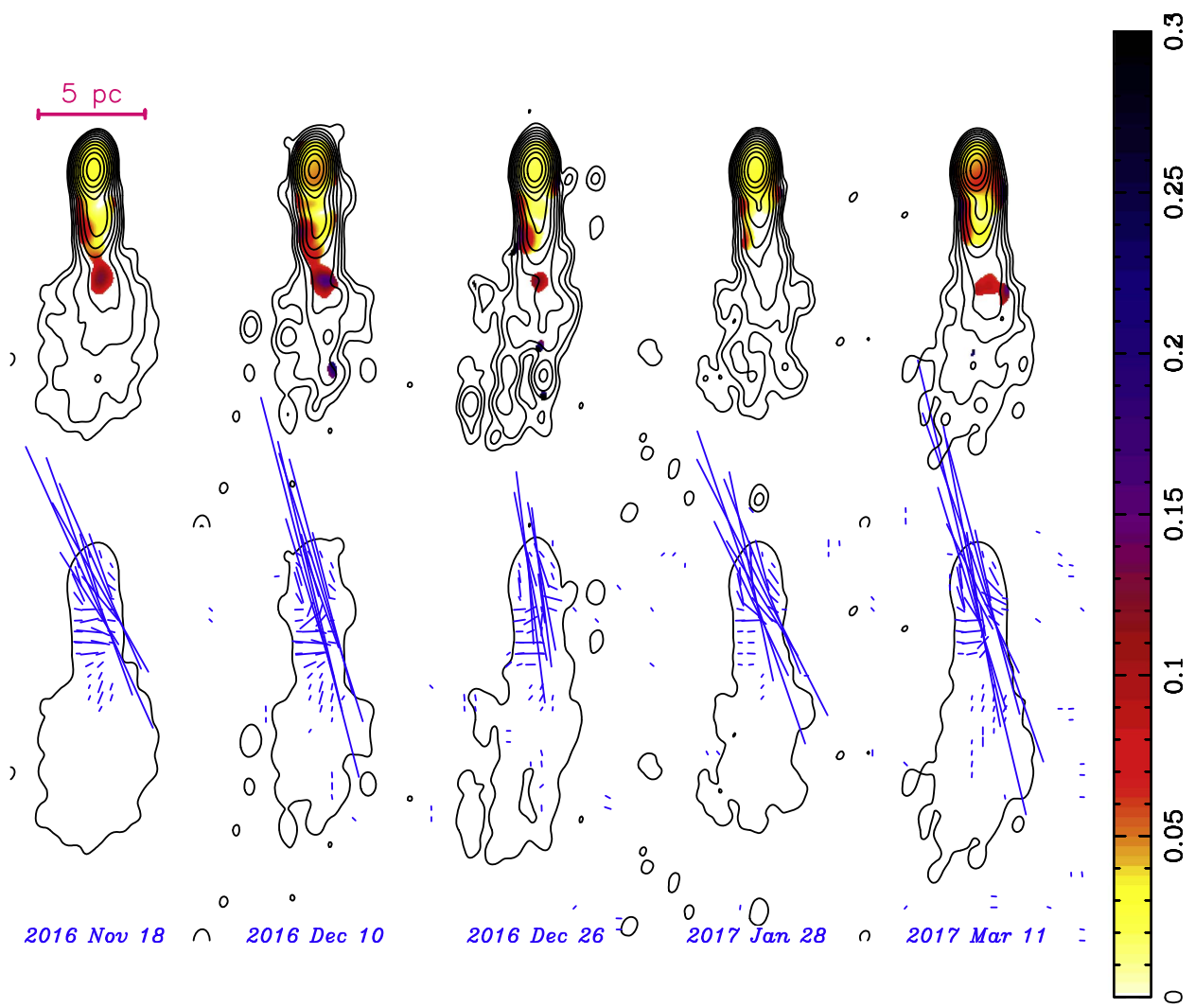

Figure 7. Images of BL Lacertae from VLBA observations at $15.4 \mathrm{GHz}$ for 10 epochs. A Gaussian restoring beam with dimensions 0.883 mas $\times 0.56$ mas and a position angle -8.2 was used. The colors in the top row of each panel show the fractional polarized level. The direction of the blue line segments in the bottom rows illustrate the EVPA, and their length corresponds to polarized intensity, the lowest of which shown is $0.5 \mathrm{mJy}^{-1}$ beam ${ }^{-1}$. The contours show the total intensity, with a base contour of $1.1 \mathrm{mJy}$ beam ${ }^{-1}$ in both top and bottom rows, and successive contours increment by factors of two in the top rows. The typical rms values of the total and polarized intensity image in these images are $0.09 \mathrm{mJy}^{\text {beam }}{ }^{-1}$ and $0.1 \mathrm{mJy}^{\mathrm{beam}}{ }^{-1}$, respectively. 

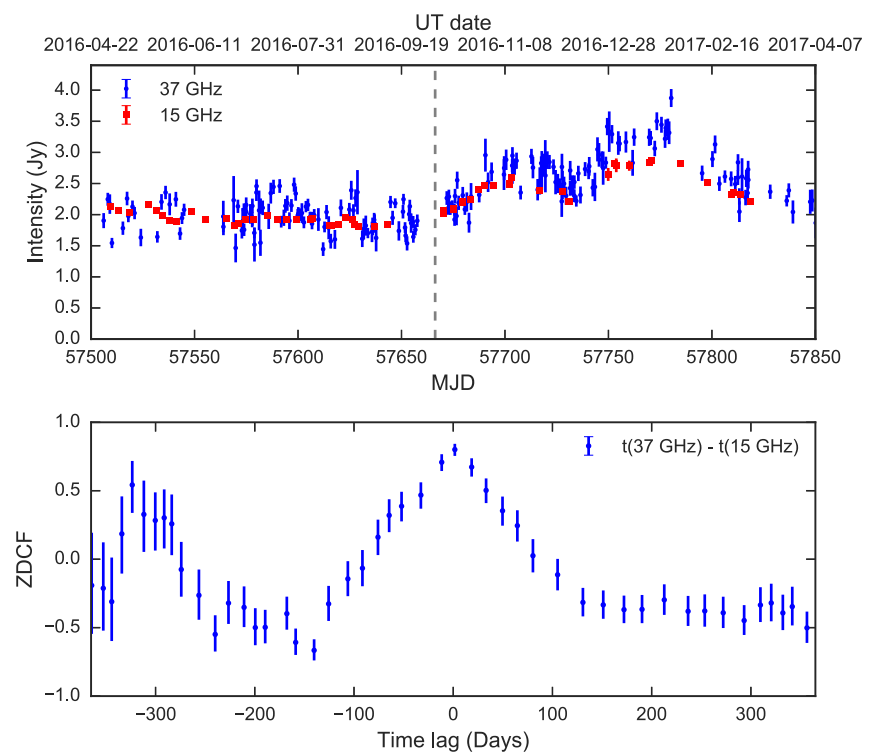

Figure 8. The top panel shows the $37 \mathrm{GHz}$ (blue dots) and $15 \mathrm{GHz}$ (red squares) radio light curves measured over $\sim 1$ yr by Metsähovi and OVRO, respectively. The gray dashed line shows the peak time of the TeV flare observed by VERITAS. The bottom panel shows the $z$-transformed discrete cross-correlation between the two light curves above. The time lag values are calculated as the difference in time $t$ between $37 \mathrm{GHz}$ and $15 \mathrm{GHz}$ so that positive time lags correspond to the $37 \mathrm{GHz}$ flux leading the $15 \mathrm{GHz}$ flux.

\subsection{On the Size of the Gamma-Ray-emitting Region}

The fastest timescale of a flare (in this case the decay time) provides a constraint on the size $R$ of the emitting region, as

$$
R \leqslant \frac{c t_{\text {decay }} \delta}{1+z}
$$

where $c$ is the speed of light, $\delta$ is the Doppler factor of the jet, ${ }^{44}$ and $z$ is the redshift of the source.

The mass of the central black hole $\left(M_{\mathrm{BH}}\right)$ of BL Lacertae was estimated to be $\sim 3.8 \times 10^{8} M_{\odot}$ by Wu et al. (2009) using the $R$-band absolute magnitude and the empirical correlation between black hole mass and bulge luminosity of the host galaxy (McLure \& Dunlop 2002). The corresponding Schwarzschild radius $R_{s}$ of the central black hole of BL Lacertae is $\sim 1.1 \times 10^{12} \mathrm{~m}\left(\sim 3.6 \times 10^{-5} \mathrm{pc}\right)$. It is worth noting that measuring the mass of a black hole is a challenging task, and $M_{\mathrm{BH}}$ values in the range $(0.16-5.01) \times 10^{8} M_{\odot}$ have been reported for BL Lacertae (see Gupta et al. 2012, and references therein).

The Doppler factor $\delta$ was estimated to be $\sim 24$ according to the method described in Hervet et al. (2016) from the propagation of a possible perturbation in the radio jet observed with VLBA at $15 \mathrm{GHz}$ (Lister et al. 2013), assuming a viewing angle of 2.2 based on radio measurements of apparent velocity. Taking the best-fit value of $t_{\text {decay }}=36_{-7}^{+8}$ minutes (see Section 2.1.1) and using Equation (5), we estimate the upper limit on the size of the emitting region to be $R \lesssim 11.9 R_{s}$.

\subsection{On the Gamma-Ray Flare Profile}

An asymmetric profile with a faster decay of the VHE gamma-ray flux was observed in the flare, which would be

\footnotetext{
$44 \delta=[\Gamma(1-\beta \cos \theta)]^{-1}$, where $\Gamma$ is the bulk Lorentz factor of the jet, and $\theta$ is the angle between the axis of the jet and the line of sight.
}

caused by an abrupt cessation of the high-energy particle injection (see, e.g., Katarzyński et al. 2003; Petropoulou et al. 2016). In this scenario, the flaring activity is attributed to fresh injection of high-energy particles into the emitting region instead of in situ acceleration of the particles. However, minimal variability in the radio band would be observable for this interpretation. Since strictly simultaneous radio observations were not performed, we cannot draw any conclusions regarding the radio variability at the time of the TeV gammaray flare. However, we note that the observed gamma-ray flare profile and the longer-term radio light curves (Figure 8) are consistent with the model proposed by Petropoulou et al. (2016). In this model, a fast gamma-ray flare can be produced by a small plasmoid in the magnetic reconnection layer, with no concurrent radio flares from the single plasmoid but a delayed radio flare powered by the entire reconnection event. The delay timescale is expected to correspond to the duration of the reconnection event, typically a few weeks. The asymmetric flare is in contrast to the more frequently observed flaring profile, a fast rise followed by a slow decay, which can be the manifestation of in situ acceleration and/or a longer cooling time (i.e., longer than the acceleration time) associated with a steep particle-energy distribution (analogous to solar flares; see, e.g., Harra et al. 2016).

BL Lacertae showed an enhancement in its GeV gamma-ray flux at the time of the TeV flare, but on a longer timescale of a few days. It also exhibited high X-ray flux on 2016 October 7 (two days after the $\mathrm{TeV}$ flare), about a factor of 10 stronger than the flux on October 6 and 8. These observations indicate efficient acceleration of relativistic particles in the jet to at least a few hundred $\mathrm{GeV}$. We note, however, that the delayed X-ray flare may or may not be related to the $\mathrm{TeV}$ gamma-ray flare, since the lack of strictly simultaneous X-ray data precludes us from ruling out the possibility of an X-ray flare simultaneous with the $\mathrm{TeV}$ gamma-ray one. The different variability timescales of the observed $\mathrm{TeV}$ and $\mathrm{GeV}$ gamma-rays give a hint that they may originate from different emitting zones. One possibility is that the $\mathrm{GeV}$ gamma-rays were produced by particles injected into and accelerated in a large shock region (e.g., a radio core; see Kovalev et al. 2009), while the $\mathrm{TeV}$ gamma-rays were produced through magnetic reconnection in a localized region (e.g., a small plasmoid in a magnetic reconnection layer, possibly at the interface between a radio core and a moving knot; see Petropoulou et al. 2016).

\subsection{On the Lorentz Factor and the Location of the Gamma-Ray-emitting Region}

Without simultaneous MWL observations with temporal resolution comparable to that of the $\mathrm{TeV}$ gamma-ray observations, we cannot construct a reliable broadband SED of the source during the $\mathrm{TeV}$ flaring state. Instead, we constrain the Lorentz factor $(\Gamma)$ of the gamma-ray-emitting region based on the gamma-ray variability, assuming two different emission mechanisms, SSC and ERC. Both models have been used to describe the broadband SED of BL Lacertae in the past (e.g., Madejski et al. 1999; Raiteri et al. 2013). However, we note that during the flare, the peak of the gamma-ray SED is located between $\sim 5 \mathrm{GeV}$ and $\sim 100 \mathrm{GeV}$, higher than that in the lower flux state (e.g., Abdo et al. 2011; Rani et al. 2013). Such behavior is most frequently observed in FSRQs and can be interpreted as an ERC process acting on IR photons in the torus region (e.g., Ghisellini \& Tavecchio 2009; Tagliaferri 
Table 2

Parameters Used to Calculate the Constraints Shown in Figure 9

\begin{tabular}{|c|c|c|c|c|c|c|c|c|c|c|c|c|c|c|}
\hline $\begin{array}{l}L_{\text {gamma }} \\
\left(\text { erg s}^{-1}\right)\end{array}$ & $\begin{array}{c}L_{\mathrm{syn}} \\
\left(\mathrm{erg} \mathrm{s}^{-1}\right)\end{array}$ & $\begin{array}{c}L_{\mathrm{d}}^{\mathrm{a}} \\
\left(\operatorname{erg~s}^{-1}\right)\end{array}$ & $\begin{array}{c}t_{\mathrm{var}} \\
(\min )\end{array}$ & $\begin{array}{l}M_{\mathrm{BH}}^{\mathrm{b}} \\
\left(M_{\odot}\right)\end{array}$ & $\begin{array}{c}E_{\text {cool }} \\
(\mathrm{TeV})\end{array}$ & $\delta / \Gamma$ & $\epsilon_{\mathrm{BLR}}$ & $\epsilon_{\mathrm{IR}}$ & $\begin{array}{c}r_{\mathrm{BLR}} \\
(\mathrm{pc})\end{array}$ & $\begin{array}{l}r_{\mathrm{IR}} \\
(\mathrm{pc})\end{array}$ & $\begin{array}{c}E_{\mathrm{BLR}} \\
(\mathrm{eV})\end{array}$ & $\begin{array}{c}E_{\mathrm{IR}} \\
(\mathrm{eV})\end{array}$ & $g_{\text {SSC }}$ & $g_{\text {ERC }}$ \\
\hline $7.8 \times 10^{45}$ & $7.8 \times 10^{44}$ & $6.0 \times 10^{44}$ & 36 & $3.8 \times 10^{8}$ & 1 & 1 & 0.1 & 0.1 & 0.025 & 0.5 & 10 & 0.3 & 0.75 & 0.5 \\
\hline
\end{tabular}

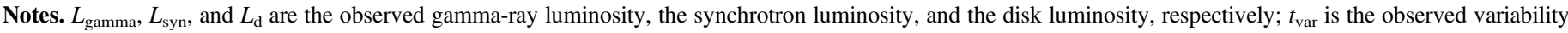

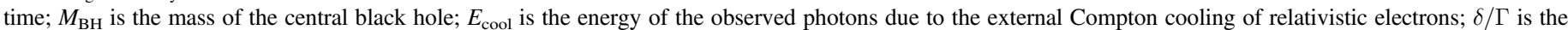

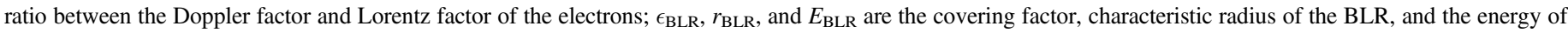

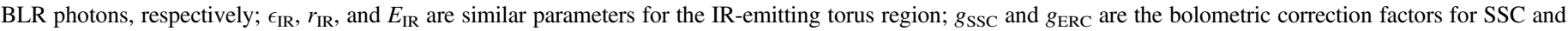
ERC mechanisms.

a Abdo et al. (2011).

${ }^{\text {b }} \mathrm{Wu}$ et al. (2009).

et al. 2015). Such an ERC process was also used to interpret the emission of BL Lacertae in a flaring state (Madejski et al. 1999; Ravasio et al. 2003).

Assuming a one-zone SSC model, we can calculate an opacity constraint on the Doppler factor $\delta$ of the TeV gammaray emitting region by requiring the pair-production optical depth to be $\leqslant 1$, following Equation (3.7) and (3.8) in Dondi \& Ghisellini (1995) (see also Arlen et al. 2013). We found that $\delta \gtrsim 13$ using the following observables: the best-fit decay time of the TeV gamma-ray flare (36 minutes), the center of the highest-energy bin with significant excess of the TeV gammaray spectrum of the source during the flare $(\sim 1.5 \mathrm{TeV})$, the $R$-band magnitude inferred from the FLWO observations on the same night (13.17), and the near-infrared spectral index (1.5; Allen et al. 1982). Assuming a viewing angle of 2.2 , the constraint $\delta \gtrsim 13$ is equivalent to a constraint on the Lorentz factor of $\Gamma \gtrsim 7$.

Assuming the gamma-rays are emitted via an ERC process, we can constrain the Lorentz factor $\Gamma$ and the distance $r$ from the central black hole of the gamma-ray-emitting region following the method described by Nalewajko et al. (2014). The collimation constraint was derived from the requirement $\Gamma \theta \lesssim 1$. Both SSC and ERC processes are considered in the calculation of the SSC constraint, while the majority of the gamma-rays are assumed to be produced via an ERC process. For the cooling constraint, only the ERC process on the thermal radiation fields close to the black hole is considered. This does not take into account any possible inverse-Compton scattering of an external synchrotron field, which, as we consider below, would loosen the cooling constraint on $\Gamma$ at large distances from the central black hole. We also assume that the emitting region is spherically symmetric. It is possible that the emitting region is not spherical (e.g., if it is passing a standing shock), and the constraints on $\Gamma$ and $r$ may change.

The values of the parameters used for the calculation of the above three constraints on $\Gamma$ and $r$ are shown in Table 2. Some of the parameters are constrained by observations, and the others are chosen so that a conservative constraint is derived. For example, we set the Compton dominance parameter $q=L_{\text {gamma }} / L_{\text {syn }}=10$ based on the observed $R$-band magnitude and the peak flux of the gamma-ray SED, the former of which should provide a good estimation of the peak of the synchrotron flux, considering that the source is a lowerfrequency-peaked BL Lac object. The SSC luminosity was set equal to the observed gamma-ray luminosity $L_{\mathrm{SSC}}=L_{\mathrm{gamma}}$ in order to obtain a conservative SSC constraint. We also used a relatively high observed gamma-ray energy $(1 \mathrm{TeV})$ for a conservative ERC cooling limit. We note that changes in the values of the parameters describing the geometry of the external radiation fields, namely the covering factor $\epsilon_{\mathrm{BLR}}$ and characteristic radius $r_{\mathrm{BLR}}$ for the BLR, and similarly $\epsilon_{\mathrm{IR}}$ and $r_{\mathrm{IR}}$ for the IR torus, which are poorly constrained by observations, could change the cooling constraint. The values of the radii used in this work are derived based on the disk luminosity $L_{\mathrm{d}}=6.0 \times 10^{44} \mathrm{erg} \mathrm{s}^{-1}$ (Abdo et al. 2011) and the relations $r_{\mathrm{BLR}}=1 \times 10^{15} \sqrt{\left(L_{\mathrm{d}} / 10^{45} \mathrm{erg} \mathrm{s}^{-1}\right)} \mathrm{m} \approx 0.025 \mathrm{pc}$ and $r_{\mathrm{IR}}=2 \times 10^{16} \sqrt{\left(L_{\mathrm{d}} / 10^{45} \mathrm{erg} \mathrm{s}^{-1}\right)} \mathrm{m} \approx 0.5 \mathrm{pc}$ (Ghisellini $\&$ Tavecchio 2015).

In this analysis, the distance $r$ between the central black hole and the gamma-ray-emitting region is constrained to be $\lesssim 12.4$ pc. If we fix the Lorentz factor at $\Gamma=24$, then we constrain the distance to be $0.01 \lesssim r / \mathrm{pc} \lesssim 0.7$. If we fix the distance at $r=1 \mathrm{pc}$, the estimated distance between the core $A O$ and the central black hole, and assume that the gamma-rays are produced as the knot $K 16$ passes the core $A 0$, then the Lorentz factor is only loosely constrained at $35 \lesssim \Gamma \lesssim 226$.

At small $r$ values $(r \lesssim 0.68 \mathrm{pc})$, the $\mathrm{SSC}$ constraint on the lower limit of $\Gamma$ is stricter than the cooling constraint. At $r=r_{\mathrm{BLR}}=0.025 \mathrm{pc}$ (the smallest distance for the VHEemitting region without heavy absorption from the radiation field in the BLR), we put a strong lower limit on the Lorentz factor $\Gamma \gtrsim 10.1$, which is larger than the archival values $(\sim 5-7)$ derived from radio observations (Jorstad et al. 2005, 2017) and consistent with the value of 24 adopted in this work. A possible explanation for the lower values of $\Gamma$ obtained from the radio observations is that they are calculated based on the apparent velocity of the superluminal features in the jet, which may travel at a lower speed than the bulk plasma flow (e.g., Lister et al. 2013; Hervet et al. 2016).

At large $r$ values $(r \gtrsim 2 \mathrm{pc}$ ), the lower limit on the Lorentz factor $\Gamma$ increases to $>100$, exceeding the typical range of $\Gamma \sim 4-50$ obtained from observations of blazars (e.g., Jorstad et al. 2005; Cohen et al. 2007; Lister et al. 2016). This indicates that another seed-photon population, such as an external synchrotron radiation field, is needed if the gamma-rayemitting region lies beyond $\sim 2$ pc.

\subsection{On the Radio and Optical Polarizations}

The 43 and $15 \mathrm{GHz}$ observations reveal that the EVPAs at the core are mostly parallel to the PA of the jet. This implies that the magnetic field is likely toroidal or strongly helical near the core, consistent with earlier observations of BL Lacertae (e.g., Gómez et al. 2016). The $15 \mathrm{GHz}$ EVPAs at larger distances away from the core become more perpendicular to the PA of the jet, indicating that the magnetic field may be more poloidal in the outer jet. Such a magnetic field configuration 


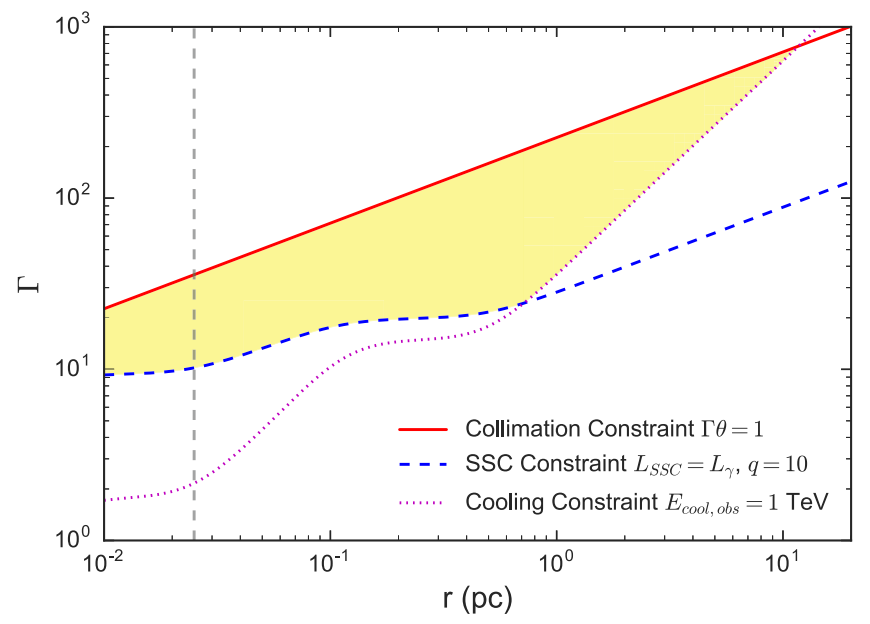

Figure 9. The constraints on the Lorentz factor $(\Gamma)$ and the distance $(r)$ between the central black hole and the gamma-ray-emitting location. The gray vertical dashed line indicates the location of the BLR $(0.025 \mathrm{pc})$ used in the calculation. The yellow shaded region illustrates the allowed parameter space.

has been proposed for low-frequency-peaked BL Lac objects (e.g., Kharb et al. 2008; Hervet et al. 2016).

Based on these radio observations, we can use the observed changes in the optical polarizations of BL Lacertae (as shown in Figure 5) to gain insights into the magnetic field structure and the location of the region that dominates the optical emission (e.g., D'arcangelo et al. 2009; Algaba et al. 2011). The optical EVPAs were observed roughly perpendicular to the PA of the jet in late 2016 September, indicating that the magnetic field is close to being aligned with the jet and likely dominated by the region downstream in the jet at that time. Similarly, the optical EVPAs became mostly parallel to the PA of the jet after late October, suggesting that the optical emission was then dominated by the core or the inner jet. We also observed the highest optical fractional polarization during this period, suggesting that the magnetic field of the core/inner jet is more ordered.

During the three days preceding the $\mathrm{TeV}$ gamma-ray flare, the optical EVPA became (temporarily) nearly aligned with the PA of the jet, but on the day before the TeV gamma-ray flare it suddenly rotated back to a direction consistent with its direction prior to this quasi-alignment. Such abrupt changes in optical polarization associated with flares are found in numerical simulations for blazars and gamma-ray bursts (Zhang et al. 2014; Deng et al. 2016), and can potentially be interpreted as resulting from the helical motion of an emitting component in a toroidal/helical magnetic field before that component reaches the shocked region (e.g., Marscher et al. 2008). However, since the fractional polarization was relatively low during this period, it is also possible that the observed change in EVPA was a random fluctuation due to a turbulent magnetic field.

A superluminal radio knot K16 was observed through a series of VLBA exposures on BL Lacertae at $43 \mathrm{GHz}$. Extrapolation of the knot position implies that the VHE gamma-ray flare happened as the knot $K 16$ crossed the quasistationary radio core. This suggests a possible association between the fast VHE gamma-ray flare and the emergence of the superluminal radio knot for the source, similar to that reported by Arlen et al. (2013).

\subsection{Interpretations of the TeV Gamma-Ray and the Radio Results}

In the model proposed by Marscher (2014), the radio core is a Mach disk at the apex of a conical shock downstream in the jet, with a transverse orientation with respect to the jet axis. When turbulent cells of plasma pass through the conical shock, relativistic electrons can be accelerated to higher energies in those cells where the magnetic field orientation relative to the shock normal is favorable. A fast gamma-ray flare can happen via inverse-Compton scattering as the relativistic plasma approaches the Mach disk at the end of the conical shock, which provides a dense source of synchrotron and SSC seed photons. After the energized plasma passes the Mach disk, a conical rarefaction causes the flow to expand and accelerate, with the bright plasma appearing as a superluminal radio knot.

In some numerical simulations, the polarization fraction drops as the magnetic field direction changes, while the EVPA can rotate owing to random fluctuation of the field or the emergence of a new field component (e.g., Marscher 2014; Zhang et al. 2014). This is consistent with the variation observed in the $R$-band polarization shortly before the VHE gamma-ray flare (see Figure 5), as well as the VLBA images at 15.4 GHz (see Figure 7). The changing superposition of the magnetic fields as the moving knot (K16) passes the quasistationary knots $(A O, A 1$, and $A 2)$ may also explain the change in the positions of $A 0, A 1$, and $A 2$ between epochs (see Figure 6).

An alternative hypothesis that can explain both the VHE gamma-ray flare and the superluminal radio knot of BL Lacertae is the breakout of a recollimation-shock zone (Hervet et al. 2016). In this model, one or more recollimation shocks, of similar nature to those in Marscher (2014) (see also Mizuno et al. 2015; Fromm et al. 2016), can form upstream in the jet where the magnetic energy density is high and appear as stationary radio knots; further downstream in the jet, particle kinetic energy becomes dominant, the magnetic field becomes unstable, and a stationary knot can be carried away by the underlying relativistic flow and become a superluminal knot. In the case of a compact region with large kinetic energy passing the recollimation-shock zone, a multi-component flare could be observed, with one component that varies slowly (i.e., on timescales of hours), thereby giving the appearance of a quasiconstant baseline in an intra-night light curve, as a result of the following sequence of events. First, in this scenario, an increase in the non-thermal emission of the shock region is expected, which leads to a flux increase on the timescale corresponding to the size of the entire shock region (as the baseline component). As the kinetic power of the jet increases in the shock zone, the magnetic field structure is subject to strong tearing instabilities, at which point a magnetic reconnection event occurs, leading to the observed fast flare. Finally, the shock zone is dragged away by the flow and enters an adiabatic expansion and cooling phase, leading to a decrease in flux and a return to the low state of the source. In the case of the 2016 flare of BL Lacertae, there is no evidence for any disruption or breakout of a stationary knot, although it is possible that the recollimation zone reformed quickly between VLBA epochs and was therefore not sampled by the observations. Therefore, future observations of flares from gamma-ray blazars, with adequate coverage after the flux decreases, can potentially reduce the ambiguity in the interpretation. 
VERITAS is supported by grants from the U.S. Department of Energy Office of Science, the U.S. National Science Foundation and the Smithsonian Institution, and by NSERC in Canada. We acknowledge the excellent work of the technical support staff at the Fred Lawrence Whipple Observatory and at the collaborating institutions in the construction and operation of the instrument.

The VERITAS Collaboration is grateful to Trevor Weekes for his seminal contributions and leadership in the field of VHE gamma-ray astrophysics, which made this study possible.

The research at Boston University was supported in part by NASA Fermi Guest Investigator Program grant 80NSSC17K 0694. The VLBA is an instrument of the Long Baseline Observatory (LBO). The LBO is a facility of the National Science Foundation operated under cooperative agreement by Associated Universities, Inc.

This research has made use of data from the MOJAVE database that is maintained by the MOJAVE team (Lister et al. 2009) and supported by NASA Fermi grant NNX15AU76G. This work made use of the Swinburne University of Technology software correlator (Deller et al. 2011), developed as part of the Australian Major National Research Facilities Programme and operated under licence. Y.Y.K. and A.B.P. are partly supported by the Russian Foundation for Basic Research (project 17-02-00197), the government of the Russian Federation (agreement 05 . Y09.21.0018), and the Alexander von Humboldt Foundation. T.S. was funded by the Academy of Finland projects 274477 and 284495.

This research has made use of data from the OVRO $40 \mathrm{~m}$ monitoring program (Richards et al. 2011) which is supported in part by NASA grants NNX08AW31G, NNX11A043G, and NNX14AQ89G and NSF grants AST-0808050 and AST1109911.

The monitoring of BL Lacertae and other blazars at the Steward Observatory is supported through NASA Fermi Guest Investigator grant NNX15AU81G.

I.A. acknowledges support by a Ramón y Cajal grant of the Ministerio de Economía y Competitividad (MINECO) of Spain. Acquisition and reduction of the MAPCAT data was supported in part by MINECO through grants AYA201014844, AYA2013-40825-P, and AYA2016-80889-P, and by the Regional Government of Andalucía through grant P09FQM-4784. The MAPCAT observations were carried out at the German-Spanish Calar Alto Observatory, which is jointly operated by the Max-Planck-Institut für Astronomie and the Instituto de Astrofísica de Andalucía-CSIC.

The St. Petersburg University team acknowledges support from Russian Science Foundation grant 17-12-01029.

Facilities: VERITAS, Fermi(LAT), Swift(XRT), SO:Kuiper, Bok, CrAO:1.25 m, CAO:2.2 m, Perkins, LX-200, FLWO:1.2 m, VLBA, Metsähovi Radio Observatory, OVRO:40m.

Software: Emcee (Foreman-Mackey et al. 2013), NumPy (van der Walt et al. 2011), Matplotlib (Hunter 2007), SciPy (Jones et al. 2001), Seaborn (Waskom et al. 2014).

\section{ORCID iDs}

W. Benbow (iD https://orcid.org/0000-0003-2098-170X

R. Bird (ib https://orcid.org/0000-0002-4596-8563

W. Cui (iD https://orcid.org/0000-0002-6324-5772

Q. Feng (i) https://orcid.org/0000-0001-6674-4238

J. P. Finley (1) https://orcid.org/0000-0002-8925-1046

A. Furniss (i) https://orcid.org/0000-0003-1614-1273
G. H. Gillanders (ib https://orcid.org/0000-0001-8763-6252

I. Gunawardhana (i) https://orcid.org/0000-0001-5583-0499

D. Hanna (i) https://orcid.org/0000-0002-8513-5603

C. A. Johnson (ib https://orcid.org/0000-0002-0641-7320

P. Kaaret (ib https://orcid.org/0000-0002-3638-0637

R. Mukherjee (i) https://orcid.org/0000-0002-3223-0754

A. N. Otte (i) https://orcid.org/0000-0002-5955-6383

M. Pohl (iD https://orcid.org/0000-0001-7861-1707

E. Pueschel (1) https://orcid.org/0000-0002-0529-1973

G. T. Richards (1) https://orcid.org/0000-0002-1408-807X

K. Shahinyan (i) https://orcid.org/0000-0001-5128-4160

S. G. Jorstad (iD https://orcid.org/0000-0001-6158-1708

A. P. Marscher (i) https://orcid.org/0000-0001-7396-3332

M. L. Lister (ib https://orcid.org/0000-0003-1315-3412

Y. Y. Kovalev (iD https://orcid.org/0000-0001-9303-3263

T. Savolainen (1D https://orcid.org/0000-0001-6214-1085

V. M. Larionov (iD https://orcid.org/0000-0002-4640-4356

M. Tornikoski (i) https://orcid.org/0000-0003-1249-6026

T. Hovatta (1) https://orcid.org/0000-0002-2024-8199

\section{References}

Abdo, A. A., Ackermann, M., Ajello, M., et al. 2010, Natur, 463, 919 Abdo, A. A., Ackermann, M., Ajello, M., et al. 2011, ApJ, 730, 101 Abeysekara, A. U., Archambault, S., Archer, A., et al. 2017, ApJ, 834, 2 Acero, F., Ackermann, M., Ajello, M., et al. 2015, ApJS, 218, 23 Ackermann, M., Ajello, M., Allafort, A., et al. 2011, ApJ, 743, 171 Aharonian, F., Akhperjanian, A. G., Bazer-Bachi, A. R., et al. 2007, ApJL, 664, L71

Albert, J., Aliu, E., Anderhub, H., et al. 2007a, ApJL, 666, L17

Albert, J., Aliu, E., Anderhub, H., et al. 2007b, ApJ, 669, 862

Aleksić, J., Antonelli, L. A., Antoranz, P., et al. 2011, ApJL, 730, L8 Alexander, T. 2013, arXiv:1302.1508

Algaba, J. C., Gabuzda, D. C., \& Smith, P. S. 2011, MNRAS, 411, 85 Allen, D. A., Ward, M. J., \& Hyland, A. R. 1982, MNRAS, 199, 969 Archambault, S., Aune, T., Behera, B., et al. 2014, ApJL, 785, L16 Arlen, T., Aune, T., Beilicke, M., et al. 2013, ApJ, 762, 92

Atwood, W., Albert, A., Baldini, L., et al. 2013, arXiv:1303.3514

Atwood, W. B., Abdo, A. A., Ackermann, M., et al. 2009, ApJ, 697, 1071

Baars, J. W. M., Genzel, R., Pauliny-Toth, I. I. K., \& Witzel, A. 1977, A\&A, 61, 99

Bach, U., Villata, M., Raiteri, C. M., et al. 2006, A\&A, 456, 105

Blandford, R. D., \& Rees, M. J. 1978, PhyS, 17, 265

Böttcher, M., \& Chiang, J. 2002, ApJ, 581, 127

Burrows, D. N., Hill, J. E., Nousek, J. A., et al. 2005, SSRv, 120, 165 Cogan, P. 2008, Proc. ICRC, 3, 1385

Cohen, M. H., Lister, M. L., Homan, D. C., et al. 2007, ApJ, 658, 232

Cohen, M. H., Meier, D. L., Arshakian, T. G., et al. 2014, ApJ, 787, 151 Daniel, M. K. 2008, Proc. ICRC, 3, 1325

D'arcangelo, F. D., Marscher, A. P., Jorstad, S. G., et al. 2009, ApJ, 697, 985 Deller, A. T., Brisken, W. F., Phillips, C. J., et al. 2011, PASP, 123, 275

Deng, W., Zhang, H., Zhang, B., \& Li, H. 2016, ApJL, 821, L12

Domínguez, A., Primack, J. R., Rosario, D. J., et al. 2011, MNRAS, 410, 2556 Dondi, L., \& Ghisellini, G. 1995, MNRAS, 273, 583

Fan, J. H., Xie, G. Z., Pecontal, E., Pecontal, A., \& Copin, Y. 1998, ApJ, 507,173

Foreman-Mackey, D., Hogg, D. W., Lang, D., \& Goodman, J. 2013, PASP, 125,306

Fromm, C. M., Perucho, M., Mimica, P., \& Ros, E. 2016, A\&A, 588, A101

Gaidos, J. A., Akerlof, C. W., Biller, S., et al. 1996, Natur, 383, 319

Gehrels, N., Chincarini, G., Giommi, P., et al. 2004, ApJ, 611, 1005

Ghisellini, G., Celotti, A., Fossati, G., Maraschi, L., \& Comastri, A. 1998, MNRAS, 301, 451

Ghisellini, G., \& Tavecchio, F. 2009, MNRAS, 397, 985

Ghisellini, G., \& Tavecchio, F. 2015, MNRAS, 448, 1060

Ghisellini, G., Tavecchio, F., \& Chiaberge, M. 2005, A\&A, 432, 401

Giannios, D., Uzdensky, D. A., \& Begelman, M. C. 2009, MNRAS, 395, L29

Gómez, J. L., Lobanov, A. P., Bruni, G., et al. 2016, ApJ, 817, 96

Gupta, S. P., Pandey, U. S., Singh, K., et al. 2012, NewA, 17, 8

Harra, L. K., Schrijver, C. J., Janvier, M., et al. 2016, SoPh, 291, 1761

Hervet, O., Boisson, C., \& Sol, H. 2015, A\&A, 578, A69 
Hervet, O., Boisson, C., \& Sol, H. 2016, A\&A, 592, A22

Holder, J. 2011, Proc. ICRC, 12, 137

Hovatta, T., Lister, M. L., Aller, M. F., et al. 2012, AJ, 144, 105

Hunter, J. D. 2007, CSE, 9, 90

Jones, E., Oliphant, T., Peterson, P., et al. 2001, SciPy: Open source scientific tools for Python, http://www.scipy.org/

Jorstad, S., \& Marscher, A. 2016, Galax, 4, 47

Jorstad, S. G., Marscher, A. P., Lister, M. L., et al. 2005, AJ, 130, 1418

Jorstad, S. G., Marscher, A. P., Morozova, D. A., et al. 2017, ApJ, 846, 98

Jorstad, S. G., Marscher, A. P., Stevens, J. A., et al. 2007, AJ, 134, 799

Kalberla, P. M. W., Burton, W. B., Hartmann, D., et al. 2005, A\&A, 440, 775

Katarzyński, K., Sol, H., \& Kus, A. 2003, A\&A, 410, 101

Kharb, P., Lister, M. L., \& Shastri, P. 2008, IJMPD, 17, 1545

Kovalev, Y. Y., Aller, H. D., Aller, M. F., et al. 2009, ApJL, 696, L17

Larson, D., Dunkley, J., Hinshaw, G., et al. 2011, ApJS, 192, 16

Lister, M. L., Aller, H. D., Aller, M. F., et al. 2009, AJ, 137, 3718

Lister, M. L., Aller, M. F., Aller, H. D., et al. 2013, AJ, 146, 120

Lister, M. L., Aller, M. F., Aller, H. D., et al. 2016, AJ, 152, 12

Madejski, G., \& Sikora, M. 2016, ARA\&A, 54, 725

Madejski, G. M., Sikora, M., Jaffe, T., et al. 1999, ApJ, 521, 145

Marscher, A. P. 2014, ApJ, 780, 87

Marscher, A. P., Jorstad, S. G., D’Arcangelo, F. D., et al. 2008, Natur, 452, 966

Max-Moerbeck, W., Hovatta, T., Richards, J. L., et al. 2014, MNRAS, 445,428

McLure, R. J., \& Dunlop, J. S. 2002, MNRAS, 331, 795
Miller, J. S., \& Hawley, S. A. 1977, ApJL, 212, L47

Mizuno, Y., Gómez, J. L., Nishikawa, K.-I., et al. 2015, ApJ, 809, 38

Moretti, A., Campana, S., Mineo, T., et al. 2005, Proc. SPIE, 5898, 360

Nalewajko, K., Begelman, M. C., \& Sikora, M. 2014, ApJ, 789, 161

Neshpor, Y. I., Chalenko, N. N., Stepanian, A. A., et al. 2001, ARep, 45, 249

Petropoulou, M., Giannios, D., \& Sironi, L. 2016, MNRAS, 462, 3325

Pollack, M., Pauls, D., \& Wiita, P. J. 2016, ApJ, 820, 12

Raiteri, C. M., Villata, M., D’Ammando, F., et al. 2013, MNRAS, 436, 1530

Rani, B., Krichbaum, T. P., Fuhrmann, L., et al. 2013, A\&A, 552, A11

Rani, B., Krichbaum, T. P., Marscher, A. P., et al. 2014, A\&A, 571, L2

Ravasio, M., Tagliaferri, G., Ghisellini, G., et al. 2003, A\&A, 408, 479

Richards, J. L., Max-Moerbeck, W., Pavlidou, V., et al. 2011, ApJS, 194, 29

Sambruna, R. M., Ghisellini, G., Hooper, E., et al. 1999, ApJ, 515, 140

Smith, J. A., Tucker, D. L., Kent, S., et al. 2002, AJ, 123, 2121

Smith, P. S., Montiel, E., Rightley, S., et al. 2009, arXiv:0912.3621

Stern, B. E., \& Poutanen, J. 2008, MNRAS, 383, 1695

Tagliaferri, G., Ghisellini, G., Perri, M., et al. 2015, ApJ, 807, 167

Teräsranta, H., Tornikoski, M., Mujunen, A., et al. 1998, A\&AS, 132, 305

van der Walt, S., Colbert, S. C., \& Varoquaux, G. 2011, CSE, 13, 22

Waskom, M., Botvinnik, O., Hobson, P., et al. 2014, seaborn: v0.5.0, Zenodo, doi: $10.5281 /$ zenodo. 12710

Wehrle, A. E., Grupe, D., Jorstad, S. G., et al. 2016, ApJ, 816, 53

Wu, Z.-Z., Gu, M.-F., \& Jiang, D.-R. 2009, RAA, 9, 168

Zhang, H., Chen, X., \& Böttcher, M. 2014, ApJ, 789, 66 